\title{
Article \\ Some properties of the basins of attraction of the Newton's method for simple nonlinear geodetic systems
}

\author{
Krzysztof Kroszczynski ${ }^{1,+\ddagger(D) 0000-0003-1197-9915}$, Damian Kiliszek ${ }^{2,+\ddagger \mathbb{D} 0000-0002-5466-9979}$ and Ireneusz \\ Winnicki $+\ddagger *$ (D0000-0001-9170-422X \\ $1 \quad$ krzysztof.kroszczynski@wat.edu.pl \\ 2 damian.kiliszek@wat.edu.pl \\ * Correspondence: ireneusz.winnicki@wat.edu.pl \\ + Current address: Faculty of Civil Engineering and Geodesy, Military University of Technology, gen. S. \\ Kaliskiego 2, 00-908 Warsaw, Poland \\ $\ddagger$ These authors contributed equally to this work.
}

\begin{abstract}
The research presented in this paper concerns the determination of the attraction basins of the Newton's iterative method which was used to solve the non-linear systems of observational equations associated with the geodetic measurements. The authors considered simple observation systems corresponding to the intersections, or linear and angular resections, used in practice. The main goal was to investigate the properties of the sets of convergent in the initial points of the applied iterative method. An important issue regarding the possibility of automatic and quick selection of such points was also considered. Therefore, the answers to the questions regarding the geometric structure of the basins, their limitations, connectedness or self-similarity were sought. The research also concerned the iterative structures of the basins, i.e. maps of the number of iterations which are necessary to achieve the convergence of the Newton's method. The determined basins were compared with the areas of convergence that result from theorems on the convergence of the Newton's method, i.e. the conditions imposed on the eigenvalues and norms of the matrices of the studied iterative systems. One of the essential results of the research is the indication that the obtained basins of attraction contain areas resulting from the theoretical premises and their diameters can be comparable with the sizes of the analyzed geodetic structures. Consequently, in the analyzed cases it is possible to construct methods that enable quick selection of the initial starting points or automation of such selection. The paper also characterizes the global convergence mechanism of the Newton's method for disconnected basins and, as a consequence, the non-local initial points, i.e. located far from the solution points.
\end{abstract}

Keywords: basin of attraction; convergence of the Newton's iterative method; planar intersection/resection.

\section{Introduction}

Determination of the coordinates of the geodetic network nodes based on the results of measurements is related with solving an optimization task. A solution to this task in terms of the least squares method is obtained by means of the iterative Newton-Gauss's method (Ghilani [3,19], Nielsen [14]). An appropriate selection of the initial values of the solution is an important element of the iterative methods $[15,19]$. Due to the process of linearization of observation equations systems, the initial point of the procedure is usually selected sufficiently close to the determined point. In the case of geodetic networks, approximate values of the network nodes coordinates are computed by means of specialized software on the basis of measurements that define the network [16-18]. In this study, the authors dealt with a more general problem of the determination of the initial approximations. The issue in question was: how far can the initial point be from the solution point (attractor) for the process to be convergent? The question is justified 
because the applied iterative method is a result of linearization while - on the other hand - the common practice indicates that the solution may quite often be determined for the initial points which do not meet the conditions of locality. Usually, in such cases the solution is found at the cost of increased number of iterations. Existence of sets of the "non-local" initial points may facilitate, for example, the automatic selection of the initial approximations of the iterative method [15]. This kind of study may also contribute to constructing practical rules of the selection. Due to the complexity of the problem resulting from multidimensionality of the studied issues (in the case of complex networks) we focused our attention on the basic geodetic constructions such as the linear and the angular intersections [3]. In this case, the solutions of the quadratic non-linear equations systems are sought after (the number of unknowns equals the number of measurements, see $[5,6])$. We noticed during the study that appropriate selection of the initial points is closely related with determination of the basins of attraction, i.e. the sets of points for which the applied iterative Newton's procedure is convergent for the discussed cases.

This paper is organized as follows. Theoretical background contains definitions and theorems used here (Section 2). Our main results that concern the study of the properties of the determined basins of attraction are presented in Sections 3 to 5 . We considered the issues of limitedness of the sets of the initial points and we characterized their geometric structures. We determined the maps of the iterations numbers that are necessary to achieve the solutions' convergence. The areas of the basins are compared with the potential areas of convergence resulting from the theorems concerning the convergence of the Newton's iterative method. Connectedness and hypothetic selfsimilarity of geometric structures and iterative basins are indicated. It is proved that appropriately quick convergence of the initial points far from the solution point is possible. Various schemes of the convergence realized on disconnected sets are also suggested. The discussion and summary of the obtained results are presented in Section 6.

\section{Theoretical background}

The study of the properties of the nonlinear systems of equations is closely related with the study of convergence of the iterative methods used to solve these systems. The following nonlinear systems of equations are considered:

$$
\mathbf{F}(\mathbf{x})=\mathbf{0}, \quad \mathbf{F}: \mathbb{R}^{n} \rightarrow \mathbb{R}^{n}
$$

where $\mathbf{F}$ is a projection (vector function) consisting of observation equations of the considered geodetic constructions - classic planar intersections or resections [11]. Except for special cases, there are no strict direct methods for solving the systems (1). It is a nontrivial issue analyzed earlier by Traub [10], Ostrowski [9], Ortega and Rheinboldt [7], [8] or Dennis Jr. and Schnabel [2]. The iterative Newton's method based on the linearization of the observation equations is used to solve:

$$
\mathbf{x}^{k+1}=\mathbf{x}^{k}-\mathbf{F}^{\prime}\left(\mathbf{x}^{k}\right)^{-1} \mathbf{F}\left(\mathbf{x}^{k}\right), \quad k=0,1, \ldots, \mathbf{x}^{0} \in B\left(\mathbf{x}^{*}\right)
$$

where the affine approximation of (2) is obtained by truncating the Taylor expansion of $\mathbf{F}$ at $\mathbf{x}^{k}$ after the linear term; $\mathbf{F}^{\prime}\left(\mathbf{x}^{k}\right)$ is the Jacobian (Fréchet derivative) of the projection $\mathbf{F}$; $\mathbf{x}^{0}$ is the initial point, and $B\left(\mathbf{x}^{*}\right)$ is the so-called "basin of attraction" for solution $\mathbf{x}^{*}$.

According to the definition, the function $\mathbf{G}: D \subset \mathbb{R}^{n} \rightarrow \mathbb{R}^{n}$ is F-differentiable at $\mathbf{x} \in D$ if for an $n \times n$ matrix $\mathbf{A}$ :

$$
\lim _{\|\mathbf{h}\| \rightarrow 0} \frac{1}{\|\mathbf{h}\|_{V}}\|\mathbf{G}(\mathbf{x}+\mathbf{h})-\mathbf{G}(\mathbf{x})-\mathbf{A} \mathbf{h}\|_{W}=0 .
$$

where: $V$ and $W$ - normed vector spaces and $\mathbf{A}$ - bounded linear operator equal to the Jacobian matrix $\mathbf{G}^{\prime}(\mathbf{x})$. 
For each solution $\mathbf{x}^{*}$ of (2) the basin of attraction $B\left(\mathbf{x}^{*}\right)$ for the iterative Newton's dynamical process $P$ (with "discrete time") can be defined as the set of all initial points $\mathbf{x}^{0}$ :

$$
B\left(\mathbf{x}^{*}\right)=\left\{\mathbf{x}^{0} \in \mathbb{R}^{n} \rightarrow\left\{\mathbf{x}^{k}\right\}=P\left(\mathbf{x}^{0}\right), \lim _{k \rightarrow \infty} \mathbf{x}^{k}=\mathbf{x}^{*}\right\}
$$

for which a (finite or infinite) $\left\{\mathbf{x}^{k}\right\}$ produced by $P: \mathbf{x}^{0} \in \mathbb{R}^{n} \rightarrow\left\{\mathbf{x}^{k}\right\} \subset \mathbb{R}^{n}$ converges to $\mathbf{x}^{*}$. The linearization of (2) induces numerous questions related to the solution's existence and its convergence:

1. How many iterations must be performed to acknowledge that the solution is achieved?

2. For what conditions and the initial points the iterations are convergent?

3. Are the sets of such points limited or unlimited?

In many cases these questions may be answered only partially. In particular, it concerns the determination of the sets of all initial points, i.e. the basins of attraction, for which the iterative process of the Newton's method is convergent.

The system (2) may be replaced by an equivalent fixed-point method system:

$$
\mathbf{x}^{k+1}=\mathbf{G}\left(\mathbf{x}^{k}\right)
$$

where the iterative function $\mathbf{G}\left(\mathbf{x}^{k}\right)=\mathbf{x}^{k}-\mathbf{F}\left(\mathbf{x}^{k}\right)^{-1} \mathbf{F}\left(\mathbf{x}^{k}\right), \mathbf{G}: \mathbb{R}^{n} \rightarrow \mathbb{R}^{n}$. Here, the point of attraction $\mathbf{x}^{*}$ refers to the fixed point $\mathbf{x}^{*}=\mathbf{G}\left(\mathbf{x}^{*}\right)$ of the iteration scheme (2). The fixed-point method is used in numerous proofs of theorems (so-called "contractionmapping theorems" and many of its variants) concerning the convergence of the iterative Newton's method (Ortega and Rheinboldt [8]).

\subsection{Selected convergence results}

Analyzing the theorems concerning the convergence of the iterative methods, two types may basically be distinguished. One type refers to the theorems of local convergence, which assume the existence of solution $\mathbf{x}^{*}$ and presence of such neighborhood $D$ of $\mathbf{x}^{*}$ that each initial point from $D$ is convergent to $\mathbf{x}^{*}$. Localness means that the initial point is appropriately close to $\mathbf{x}^{*}$ : e.g. see Newton's Attraction Theorem (Ortega and Rheinboldt [8]). For the theorems of other type the existence of solution $\mathbf{x}^{*}$ is not assumed, but it is shown that for some conditions enforced on the projection $\mathbf{F}$ and the initial points set of the Newton's method, there is a solution to which the iterative process is convergent, like Contraction-Mapping Theorem or Newton-Kantorovich Theorem (Ortega and Rheinboldt [8]). It would be ideal if the Newton's method was convergent for every initial point belonging to the area of the projection domain $\mathbf{F}$. Within this research this case of the linear intersection is studied. Global convergence of iterative methods is rare. Typical assumptions in the theorems, usually very rigorous, guarantee not much more than local convergence. In order to illustrate the problem, the theorems of the mentioned types may be quoted. The first one is the Ostrowski's theorem (Ostrowski [9] and Ortega and Rheinboldt [8]), which gives the sufficient conditions for a solution of $\mathbf{F}(\mathbf{x})=0$ to be a point of the attraction.

Theorem 1 (Ostrowski [9]). Suppose, that $\mathbf{G}(\mathbf{x})=\mathbf{x}-\mathbf{F}^{\prime}(\mathbf{x})^{-1} \mathbf{F}(\mathbf{x}): D \subset \mathbb{R}^{n} \rightarrow \mathbb{R}^{n}$ has a fixed point $\mathbf{x}^{*} \in D$ where $\mathbf{G}(\mathbf{x})$ is F-differentiable at $\mathbf{x}^{*}$. If the spectral radius $\lambda=$ $\rho\left(\mathbf{G}^{\prime}\left(\mathbf{x}^{*}\right)\right)<1$ then $\mathbf{x}^{*}$ is a point of attraction of the fixed-point iteration (5) and simultaneously of the Newton's iteration scheme (2).

In this theorem the existence of $\mathbf{x}^{*}$ had to be assumed. Moreover, only local instead of global convergence is guaranteed, and merely the sufficiency, but not the necessity, of the condition is asserted (Ortega and Rheinboldt [8]). Differentiability of $\mathbf{G}(\mathbf{x})$ at $\mathbf{x}^{*}$ results in the existence of the neighborhood of $\mathbf{x}^{*}\left(S=S\left(\mathbf{x}^{*}, \delta\right) \subset D, \delta>0\right)$ that 
is a set of the initial points for which the iterative Newton's method is convergent. Presented research indicates that the neighborhood is usually defined by a small value of the parameter $\delta$ and, because of this, it is usually only a part of the basin of attraction. Dennis and Schnabel [2] indicated that the radius of the Newton's method convergence is inversely proportional to the relative nonlinearity of $\mathbf{F}(\mathbf{x})$ at $\mathbf{x}^{*}$. They suggested that the relative nonlinearity of a function is the key factor determining the behavior of the iterative algorithms, and all convergence theorems could be restarted and proven in terms of this concept. It was illustrated in details in [2] (p. 92). Dennis and Schnabel estimated how far the region of quadratic convergence extends in the direction from $\mathbf{x}^{*}$ in which $\mathbf{F}(\mathbf{x})$ is the most nonlinear. On the other hand, the region of convergence of the Newton's method may be much larger in a direction of less nonlinearity of $\mathbf{F}(\mathbf{x})$. Among other things, for this reason the basins of attraction must not be considered equivalent with areas of convergence resulting from the assumptions of the theorems concerning the convergence of the Newton's method.

The other type of theorems is related with the Banach fixed-point theorem [1], also known as the Contraction-Mapping Theorem. This classical theorem considers any iterative method of the form $\mathbf{x}^{k}=\mathbf{G}\left(\mathbf{x}^{k}\right)$ and states conditions for $\mathbf{G}(\mathbf{x})$, under which the sequence $\left\{\mathbf{x}^{k}\right\}$ converges to a point $\mathbf{x}^{*}$ from any point $\mathbf{x}^{0}$ in a region $D$. Furthermore, $\mathbf{x}^{*}$ is shown to be the unique point in $D$, such that $\mathbf{G}\left(\mathbf{x}^{*}\right)=\mathbf{x}^{*}$. The Contraction-Mapping Theorem is broader (but weaker due to speed of the convergence) than the Ostrowski's Theorem or the above mentioned Newton-Kantorovich Theorem (Dennis and Schnabel [2]). In the theorem presented below, which is a version of the Contraction-Mapping Theorem (Ortega and Rheinboldt [8]), it is characteristic that neither the existence of a solution $\mathbf{x}^{*}$, nor a local convergence $\left(\mathbf{x}^{0}\right.$ is sufficiently close to $\left.\mathbf{x}^{*}\right)$, are assumed.

Theorem 2 (Ortega and Rheinboldt [8]). Suppose that $\mathbf{G}: D \subset \mathbb{R}^{n} \rightarrow \mathbb{R}^{n}$ maps a closed set $S \subset D$ into itself and that there exists $p \geq 1$, and a constant $\lambda \in(0,1)$ such that:

$$
\left\|\mathbf{G}^{p}(\mathbf{y})-\mathbf{G}^{p}(\mathbf{x})\right\|<\lambda\|\mathbf{y}-\mathbf{x}\|, \quad \forall \mathbf{x}, \mathbf{y} \in S
$$

Then $\mathbf{G}$ has a unique fixed point $\mathbf{x}^{*}=\mathbf{G}\left(\mathbf{x}^{*}\right)$ in $S$ and for any $\mathbf{x}^{0} \in S$ the iterations converge to and satisfy:

$$
\left\|\mathbf{x}^{k}-\mathbf{x}^{*}\right\|<\frac{\lambda^{k}}{1-\lambda}\left\|\mathbf{x}^{1}-\mathbf{x}^{0}\right\|, \quad k \geq 0 .
$$

This theorem can be used to test whether there is any region $D$ such that the points generated by $\mathbf{x}^{k}=\mathbf{G}\left(\mathbf{x}^{k}\right)$ from an $\mathbf{x}^{0} \in S$ will converge to the root of $\mathbf{F}(\mathbf{x})$. Furthermore, the theorem indicates that if is found an area $D$, in which the theorem contraction condition is satisfied, then $D$ is a basin of attraction. Since we do not assume local convergence, a study of convergence for the initial points far from the solution $\mathbf{x}^{*}$ may also be considered. There is a certain disparity here, because the Newton's method, due to the expansion into truncated Taylor series, is a local procedure. It may be noticed that theorems of this type theoretically permit wider range of search for basins of attraction. We observe it in the case of the considered here basins of attraction of linear and angular intersections and combined linear intersection and angular resection. It is possible to analytically confirm some of the geometric properties of these sets, e.g. in the case of the existing symmetry of the intersections. Then, global contraction is possible on all $\mathbb{R}^{n}-$ the attraction set is a space or subspace of $\mathbb{R}^{n}$. More complex cases which cannot be dealt with by means of analytical methods, must be analyzed using numerical computations, especially because there are sets of initial points $\mathbf{x}^{0}$ distant from $\mathbf{x}^{*}$ and convergent to the solution $\mathbf{x}^{*}$, which do not satisfy the contraction condition and the conditions of local theorems. 


\subsection{The Newton's method algorithm}

In order to determine the vector of the solution $\mathbf{x}^{k+1}$ for the subsequent iteration step $k+1$ using the relations (2), the following system of the linear equations is solved:

$$
\mathbf{F}^{\prime}\left(\mathbf{x}^{k}\right) \Delta \mathbf{x}=-\mathbf{F}\left(\mathbf{x}^{k}\right), \quad \Delta \mathbf{x}=\mathbf{x}^{k+1}-\mathbf{x}^{k} \rightarrow \mathbf{x}^{k+1}=\mathbf{x}^{k}+\Delta \mathbf{x}, k=0,1, \ldots
$$

This approach enables to avoid the inverse Jacobian $\mathbf{F}^{\prime}\left(\mathbf{x}^{k}\right)$ computation. The criteria of completing the computations are based on the conditions under which the function $\mathbf{F}\left(\mathbf{x}^{k}\right)$ achieves components values sufficiently close to zero:

$$
\mathbf{F}\left(\mathbf{x}^{k}\right) \leq \varepsilon_{F}
$$

and sufficiently small differences of distance (norm) between the subsequent vectors of iteration:

$$
\left\|\mathbf{x}^{k+1}-\mathbf{x}^{k}\right\| \leq \varepsilon_{x} .
$$

In practice, the parameters $\varepsilon_{F}$ and $\varepsilon_{x}$ are related with the uncertainty of measurements. The machine precision is often assumed for the values of $\varepsilon_{F}$ and $\varepsilon_{x}$, e.g. the relative spacing between any two adjacent numbers in the machine's floating point system. Another type of the condition is related to the permissible number of iterations. It enables to stop the computations in case of solution divergence. In general, the number of iterations depends on the type of convergence of the iterative algorithm. For standard assumptions concerning function $\mathbf{F}(\mathbf{x})$ when the initial point $\mathbf{x}^{0}$ is sufficiently close to $\mathbf{x}^{*}$, the Newton iteration scheme converges at a quadratic rate to the solution: $\mathbf{x}^{k} \rightarrow \mathbf{x}^{*}$ (see Kelley [5]). Quadratic convergence means that the distance between the subsequent approximations and the precise solution $\mathbf{x}^{*}$ decrease according to the following relation:

$$
\left\|\mathbf{x}^{k+1}-\mathbf{x}^{*}\right\|<C\left\|\mathbf{x}^{k}-\mathbf{x}^{*}\right\|, C>0
$$

This property is important because a small number of iterations is required to achieve a prescribed accuracy in the computations. Generally, there are various types of convergences (Ortega and Rheinboldt [8]). In numerous cases considered in the research, at least linear convergence is found when the initial points are far away from the solution point:

$$
\left\|\mathbf{x}^{k+1}-\mathbf{x}^{*}\right\|<\alpha\left\|\mathbf{x}^{k}-\mathbf{x}^{*}\right\|, 0 \leq \alpha \leq 1
$$

The case when $\alpha \approx 1$, it is unacceptably slow.

\subsection{Methods of constructing the basins of attraction}

Both the analytical and the practical approach to analyzing the properties of the attraction basins $B$ is discussed in [12]. In the practical approach to determining the basins, the areas $D$ belonging to the domain of the function $\mathbf{F}(\mathbf{x})$ are covered by regular grids of various sizes and spatial resolutions. The nodes of the grids are the initial points $\mathbf{x}^{0}$ of the Newton's method. The convergence of the method for $\mathbf{x}^{0} \in D$ means that $\mathbf{x}^{0}$ belongs to the basin of attraction $B$. This way of grid generation was used e.g. for presenting the map of the number of iterations required to achieve the solution $\mathbf{x}^{*}$. In the case studies of basins' boundaries and basins' finiteness related with very extent areas, randomly generated sets of the initial points $\mathbf{x}^{0}$ are used:

$$
\left(x_{i}^{0}, y_{j}^{0}\right)=x_{\min }+\left(x_{\max }-x_{\min }\right) \cdot \psi_{i}, y_{\min }+\left(y_{\max }-y_{\min }\right) \cdot \zeta_{j}, i=1, \ldots, n, j=1, \ldots, m
$$

where $x_{\min }, x_{\max }, y_{\min }, y_{\max }$ denote values defining the size of the basin, and $\psi_{i}, \zeta_{j}$ are pseudorandom numbers of uniform distribution. 
For studying the Newton's method convergence the balls of the random numbers are generated by the following relations:

$$
x_{i}=x_{0}+r_{i} \cdot \sin \left(t_{i}\right), \quad y_{i}=y_{0}+r_{i} \cdot \cos \left(t_{i}\right), \quad t=2 \pi \cdot \xi_{i}, r_{i}=\sqrt{\psi_{i}}, \quad i=1, \ldots, n
$$

where: $\left(x_{0}, y_{0}\right)$ - the coordinates of the ball center.

\section{Linear intersection}

The linear intersection (Figure 1 ) is designed to estimate the position of an unknown point $\mathrm{C}(x, y)$ using two distances $a$ and $b$ measured from two known points $\mathrm{A}$ and $\mathrm{B}$. In order to simplify the calculations, it was assumed that the intersection base of the length of $c$ coincides with the $y$ axis of the geodetic coordinate frame.

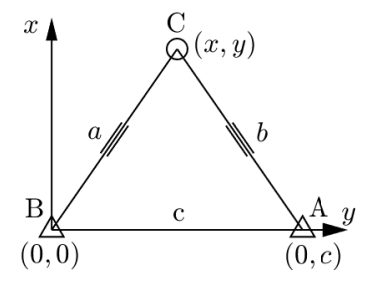

Figure 1. Linear intersection.

Two non-equivalent - with respect to the iterative methods - systems of observation equations for linear intersection are considered: polynomial (15) and radical (16) which in the assumed coordinate system are written as follows:

$$
\begin{gathered}
\mathbf{F}(\mathbf{x})=\left[\begin{array}{l}
f_{1}(x, y)=x^{2}+y^{2}-a^{2}=0 \\
f_{2}(x, y)=x^{2}+(c-y)^{2}-b^{2}=0
\end{array}\right] \\
\mathbf{F}(\mathbf{x})=\left[\begin{array}{l}
f_{1}(x, y)=\sqrt{x^{2}+y^{2}}-a=0 \\
f_{2}(x, y)=\sqrt{x^{2}+(c-y)^{2}}-b=0
\end{array}\right]
\end{gathered}
$$

where $a$ and $b$ are the measured distances, $c$ is the known length of the intersection base and $y$ is the coordinate of point A.

The equations systems (15) and (16) are invariant with respect to translation vector $\left[t_{x}, t_{y}\right]$ and isotropic scaling $s(s-$ scale coefficient), i.e. the replacement of coordinates: $x^{\prime}=t_{x}+s \cdot x, y^{\prime}=t_{y}+s \cdot y, c^{\prime}=t_{y}+s \cdot c$ and measurements: $a^{\prime}=s \cdot a, b^{\prime}=s \cdot b$. For this reason, the geometric properties of the basins of attraction of the systems subjected to such transformations are the same. An example of a studied subfamily of intersections: $(0<a<2,0<b<1, c=1)$ belonging to the family defined by the inequality: $0<a<2,0<b<2,0<c<2$ is presented in Figure 2 .

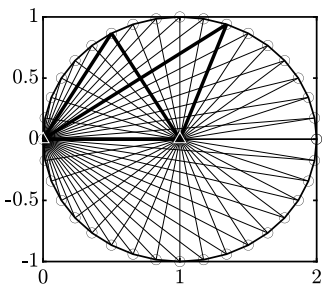

Figure 2. Subfamily of linear intersections.

The exact solution $\left(x^{*}, y^{*}\right)$ of the systems (15) and (16) in the assumed coordinate system is given by the following relations (see Appendix A): 


$$
x^{*}= \pm \frac{\sqrt{(a+b-c)(a+b+c)(c+b-a)(c+a-b)}}{2 c}, y^{*}=\frac{a^{2}-b^{2}+c^{2}}{2 c}
$$

The expression in the root in (17) is positive, which results from the triangle inequalities: $a+b-c>0, a+b+c>0, c+b-a>0$ and $c+a-b>0$. This study is limited to the case of positive solutions: $x>0$.

\subsection{Polynomial variant of the linear intersection}

For studying the basin of attraction of the linear intersection (15), the following iterative function of the Newton's method is used (the superscripts $k$ are omitted here):

$$
\mathbf{G}(\mathbf{x})=\mathbf{x}-\mathbf{F}^{\prime}(\mathbf{x})^{-1} \mathbf{F}(\mathbf{x})=\underbrace{\left[\begin{array}{l}
x \\
y
\end{array}\right]}_{\mathbf{x}}-\underbrace{\frac{1}{2 c} \cdot\left[\begin{array}{cc}
(c-y) / x & y / x \\
1 & -1
\end{array}\right]}_{\mathbf{F}^{\prime}(\mathbf{x})^{-1}} \cdot \underbrace{\left[\begin{array}{c}
x^{2}+y^{2}-a^{2} \\
x^{2}+(c-y)^{2}-b^{2}
\end{array}\right]}_{\left[f_{1} f_{2}\right]^{T}}
$$

where $\mathbf{F}^{\prime}(\mathbf{x})^{-1}$ is the reciprocal of the Jacobian matrix $\mathbf{F}^{\prime}(\mathbf{x})$ of the projection $\mathbf{F}(\mathbf{x})$ (15). The matrices $\mathbf{F}^{\prime}(\mathbf{x})$ and $\mathbf{F}^{\prime}(\mathbf{x})^{-1}$ are singular in points coinciding with the $y$ axis of the coordinate system (Figure 1). Expanding (18) and applying $y^{*}$ from (17) we obtain:

$$
\mathbf{G}(\mathbf{x})=\left[\begin{array}{c}
g\left(x, y^{*}\right) \\
\left(a^{2}-b^{2}+c^{2}\right) /(2 c)
\end{array}\right]=\left[\begin{array}{c}
g(x) \\
y^{*}
\end{array}\right]
$$

Relation (19) indicates that $\mathbf{G}(\mathbf{x})$ is a function of the coordinate $x$ only. It means that independently of the value of $x$ after the first iteration, the value of $y$ is constant and equal to $y^{*}$. The solution is hence sought after along a straight line perpendicular to $y$ at $y^{*}$. After substituting $y^{*}$ with $g\left(x, y^{*}\right)$, after elementary conversion (see Appendix B) we obtain:

$$
g(x)=\frac{x}{2}+\frac{(a+b-c)(a+b+c)(c+b-a)(c+a-b)}{8 c^{2} x}>0, \text { for } x>0
$$

Including the exact solution $x^{*}(17)$ in (20) we may rewrite it as an iterative function of the root of the quadratic equation $x^{2}=c, c=\left(x^{*}\right)^{2}$ :

$$
x=g(x)=x / 2+\left(x^{*}\right)^{2} /(2 x)>0, \text { for } x>0
$$

Relation (21) indicates that after the first iteration, for any initial point of the coordinate $x^{0}>0$, we obtain a point of the coordinate $x=g\left(x^{0}\right)>x^{*}$. This remark is due to the inequality:

$$
x=g(x)=x / 2+\left(x^{*}\right)^{2} /(2 x)>x^{*}, x>0
$$

which for $x>0$ is equivalent to the following true inequality

$$
x^{2}-2 x x^{*}+\left(x^{*}\right)^{2}=\left(x-x^{*}\right)^{2}>0, \text { for } x \neq x^{*}
$$

for every $x \neq x^{*}$.

This is illustrated in Figure 3(a) where the balls $K\left(\mathbf{x}^{0}\right)$ of the randomly generated initial points $\mathbf{x}^{0}$ are converted into line segments coinciding with the straight line $y=y^{*}$, which coordinates $x$ satisfy the condition: $x>x^{*}$. This result is essential in the study of the boundaries of the basin of attraction of the polynomial linear intersection.

Considering (21) we may notice that in the case when the initial value of the coordinate approaches the $y$ axis, i.e. $x^{0} \rightarrow 0^{+}$, then $x^{1}=g\left(x^{0}\right) \rightarrow+\infty$. Consequently, 
(a)

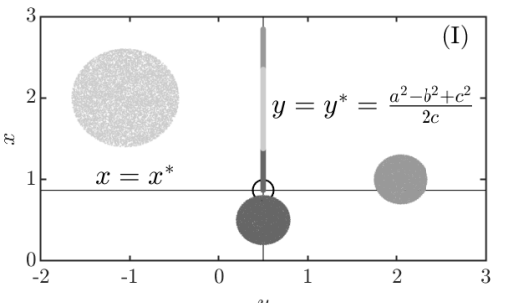

(b)

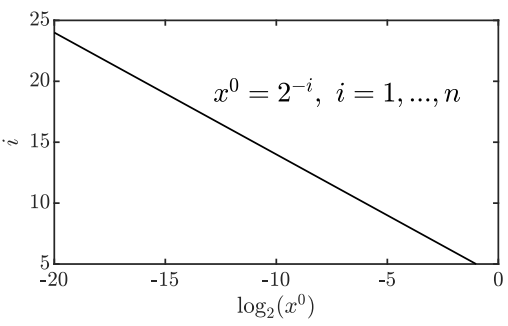

Figure 3. (a) - projection of the balls of the initial points after the first iteration of the Newton's method, (b) - number of iterations for $x^{0} \rightarrow 0^{+}$.

the number of iterations as a function of: $\log _{2}\left(x^{0}\right), x^{0}=2^{-i}, i=1, n$ also increases (see Figure 3(b)). This indicates that the number increases approximately exponentially with decreasing $x^{0}=2^{-i}, i=1, n$. This effect can be seen in Figure 4 where at the lower parts of the plots, the contour lines presenting the numbers of iterations are packed together.

Determining the derivative $g^{\prime}(x)$ :

$$
g^{\prime}(x)=1 / 2-\left(x^{*}\right)^{2} /\left(2 x^{2}\right), \text { for } x>x^{*}
$$

and including the inequality (21) (satisfied already after the first iteration), we may notice that:

$$
-1 / 2<g^{\prime}(x)<1 / 2,\left(0<\left(x^{*}\right)^{2} / x^{2}<1, \lim _{x \rightarrow \infty} g^{\prime}(x)=1 / 2,\right.
$$

Relation (23) indicates that projection $g(x)$ is contractive for every $x>0$. Determining the derivative $\mathbf{G}^{\prime}(\mathbf{x})$ of projection (19) we obtain:

$$
\mathbf{G}^{\prime}(\mathbf{x})=\left[\begin{array}{cc}
g^{\prime}(x) & 0 \\
0 & 0
\end{array}\right]
$$

Relation (24) indicates that $\lambda_{x}=g^{\prime}(x)$ is the non-zero eigenvalue of matrix $\mathbf{G}^{\prime}(\mathbf{x})$. According to (23), $\lambda_{x}=\left|g^{\prime}(x)\right|<1 / 2$, which means that $\mathbf{G}(\mathbf{x})$ is a contractive projection in the half-plane: $x>0$ which includes the intersected point.
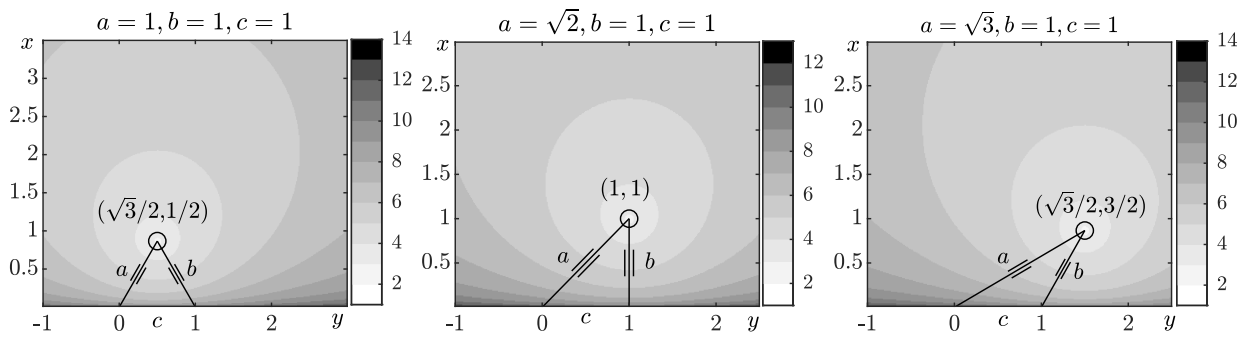

Figure 4. Fragments of the basins of attraction of the polynomial variant of the linear intersections including the iterative structure (for $c=1$ and $\varepsilon=10^{-6}$ ).

The fragments of the attraction basins for various linear intersections presented in (18) are obtained by covering the area of intersections with a regular grid, which nodes are the initial points $\mathbf{x}^{0}=\left(x^{0}, y^{0}\right)$ of the Newton's method. The numbers of iterations (gray-scale bar legend in Figure 4) - necessary to achieve the solution $\left(x^{*}, y^{*}\right)$ for the assumed accuracy $\varepsilon\left(\varepsilon_{x}, \varepsilon_{F}\right)$ - are determined for each of the points. The obtained results indicate that the basin of attraction of the Newton's method for the polynomial variant of the linear intersection, given with (15), is theoretically unlimited. Regardless of the shape of the intersection construction, which is defined by the measurements $a$ and $b$, it is the half-plane $x>0$ that includes the intersected point $\left(x^{*}, y^{*}\right)$. In the case of $\left(x^{*}<0, y^{*}\right)$, the basin of attraction is a symmetric reflection of the basin for $x>0$. 


\subsection{Radical variant of the symmetric linear intersection}

For studying the basins of attraction (16), the following iterative Newton's function is used:

$$
\mathbf{G}(\mathbf{x})=\underbrace{\left[\begin{array}{l}
x \\
y
\end{array}\right]}_{\mathbf{x}}-\underbrace{\left[\begin{array}{rr}
(c-y) \sqrt{x^{2}+y^{2}} / x & y \sqrt{x^{2}+(c-y)^{2}} / x \\
\sqrt{x^{2}+y^{2}} & -\sqrt{x^{2}+(c-y)^{2}}
\end{array}\right]}_{\mathbf{F}^{\prime}(\mathbf{x})^{-1}} \cdot \underbrace{\left[\begin{array}{c}
\sqrt{x^{2}+y^{2}}-a \\
\sqrt{x^{2}+(c-y)^{2}}-b
\end{array}\right]}_{\left[f_{1} f_{2}\right]^{T}}
$$

Similarly as for the intersection (15), the matrices $\mathbf{F}^{\prime}(\mathbf{x})$ and $\mathbf{F}^{\prime}(\mathbf{x})^{-1}$ are singular for the initial points $\left(x^{0}, y^{0}\right)$ coinciding with the $y$ axis, i.e. on the straight line given by $x=0$, especially when the initial points $\mathbf{x}^{0}$ coincide with the points that determine the intersection base. Figure 5(a) presents fragments of the symmetric basin of attraction for the symmetric intersection: $a=b=c=1$. It can be seen that there are nonlocal neighborhoods (of radiuses comparable to the intersection base length) for which the initial points are convergent after a few ( 3 or 4$)$ iterations. Figures $5(b)$ and (c) illustrate that decreasing value of $\varepsilon\left(\varepsilon_{x}, \varepsilon_{F}\right)$ changes the iterative structure of the basin (smaller values of $\varepsilon$ correspond to larger numbers of iterations). However, $\varepsilon$ does not impact the global geometric structure of the basin of attraction. Differentiability of the function (25) and the quadratic convergence of the Newton's method in an appropriately small neighborhood of $\mathbf{x}^{*}$, indicate that if the initial point is convergent for $\varepsilon_{1}$, it is also convergent for $\varepsilon_{2}<\varepsilon_{1}$.

a) -2
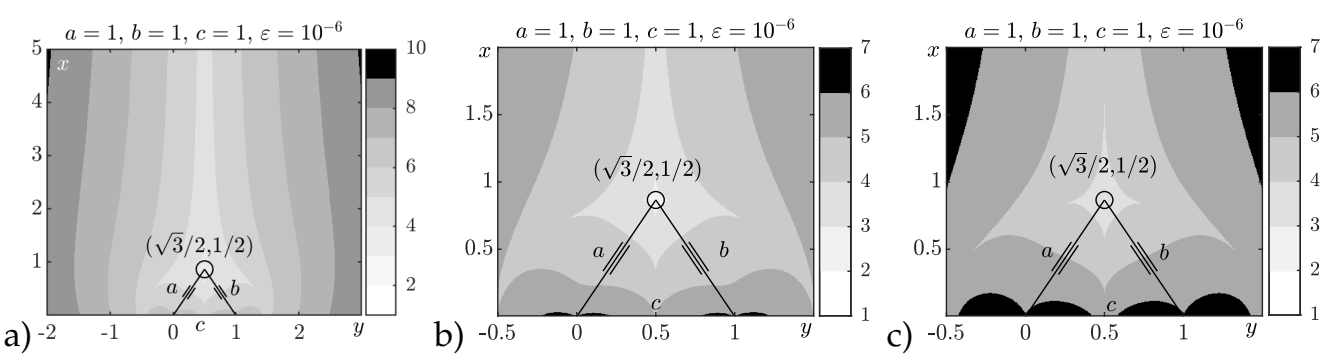

Figure 5. Fragment of the basin of attraction of the symmetric intersection and its magnifications for $\varepsilon_{x}=\varepsilon_{F}=10^{-6}, 10^{-9}$.

The case of the symmetric intersection, subjectable to analytic approach, is considered during the study of the basic properties of the basin of attraction of the linear intersection (25). The exact solution of the intersection is the point of the coordinates: $x^{*}=\frac{\sqrt{3}}{2}, y^{*}=\frac{1}{2}$. Taking into account the symmetry of the basin of attraction in Figure 5 , a fragment of the straight line $y^{*}=\frac{1}{2}$ was assumed as the set of the initial points. Including the equations of the observation system (16) in (25), the component $g(x)$ (see Figure $6(\mathrm{a})$ ) of the iterative function $\mathbf{G}(\mathbf{x})$ of the Newton's method was determined as:

$$
\begin{gathered}
\mathbf{x}=\mathbf{G}(\mathbf{x})=\left[\begin{array}{c}
g(x) \\
y^{*}=\frac{1}{2}
\end{array}\right] \\
g(x)=\left(\sqrt{x^{2}+\frac{1}{4}}-\frac{1}{4}\right) / x, g(x)^{\prime}=1 / \sqrt{x^{2}+\frac{1}{4}}+\left(\frac{1}{4}-\sqrt{x^{2}+\frac{1}{4}}\right) / x^{2}
\end{gathered} .
$$

Function $g(x)$ has its minimum $\left(g^{\prime}\left(x=\frac{\sqrt{3}}{3}\right)=0, g^{\prime \prime}\left(x=\frac{\sqrt{3}}{3}\right)>0\right)$ at point $x^{*}=\frac{\sqrt{3}}{2}$ with the value of $g\left(\frac{\sqrt{3}}{2}\right)=\frac{\sqrt{3}}{2}$ (Figure 6(b)). For this reason, for every initial value $x^{0} \neq x^{*}$, we have $x=g\left(x^{0}\right)>x^{*}$. The condition: $0<\lambda=g^{\prime}(x)<1$ is satisfied in the range $x \in\left(\frac{\sqrt{3}}{2}, \infty\right)$ because the maximum value of the derivative of the projection satisfies the condition: $g^{\prime}(x)<1$ and the limit value: $\lim _{x \rightarrow \infty} g^{\prime}(x)=0$. It means that the 
(a)
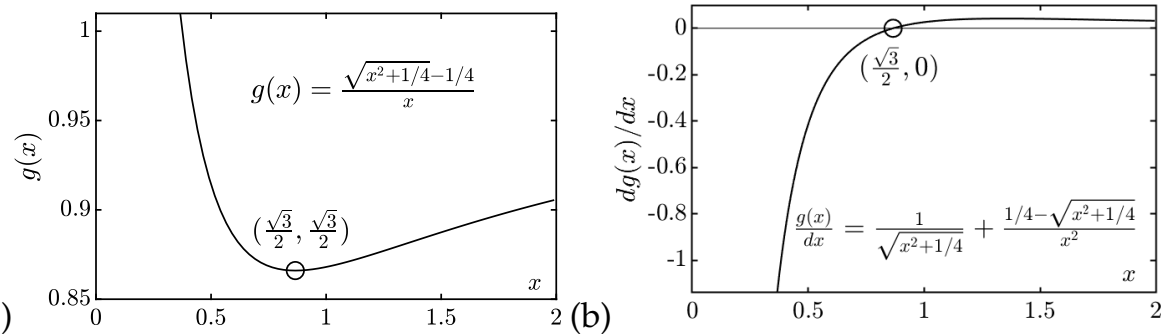

Figure 6. Component $g(x)$ of the iterative function of the Newton's method.

projection (26) is contractive on the straight line $y^{*}=\frac{1}{2}$. In general, without limitation to the case presented in Figure 7(a) it may be noticed (Figure 7(b) and (c)) that the balls of the initial points of $\left(y \neq y^{*}\right)$ are projected into similar areas (resembling the profiles of the Zukowski's wings). The areas are located above the straight line defined by the equation: $x=x^{*}=\sqrt{3} / 2$. In subsequent iterations they are converged to an area that includes point $x^{*}$ accepted as the intersection solution.
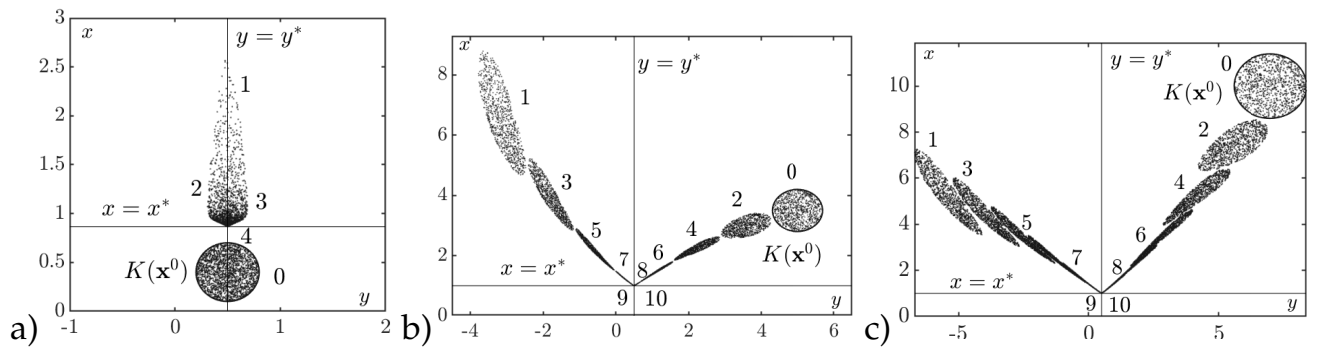

Figure 7. Projection of the balls of the random initial points for the Newton's iterations.

The obtained results signify that the basin of attraction of the symmetric intersection is (theoretically) unlimited on the straight line $y=y^{*}$. The symmetry of the set does not, however, clarify what it is like in other directions. Analytical calculations related to e.g. the direction perpendicular to the straight line $y=y^{*}$ are much more complicated. It is because, unlike for the polynomial intersection (15), the convergence mechanism (Figures 7(b) and (c)) is entirely different. The method of the least squares is applied to assess the number of iterations necessary for determining the basin of attraction of a given width $y= \pm 2^{j}, j=-1,0, \ldots, l$, measured along the straight lines defined by equations: $x=2^{k}, k=-m, \ldots, 0, \ldots, n$.
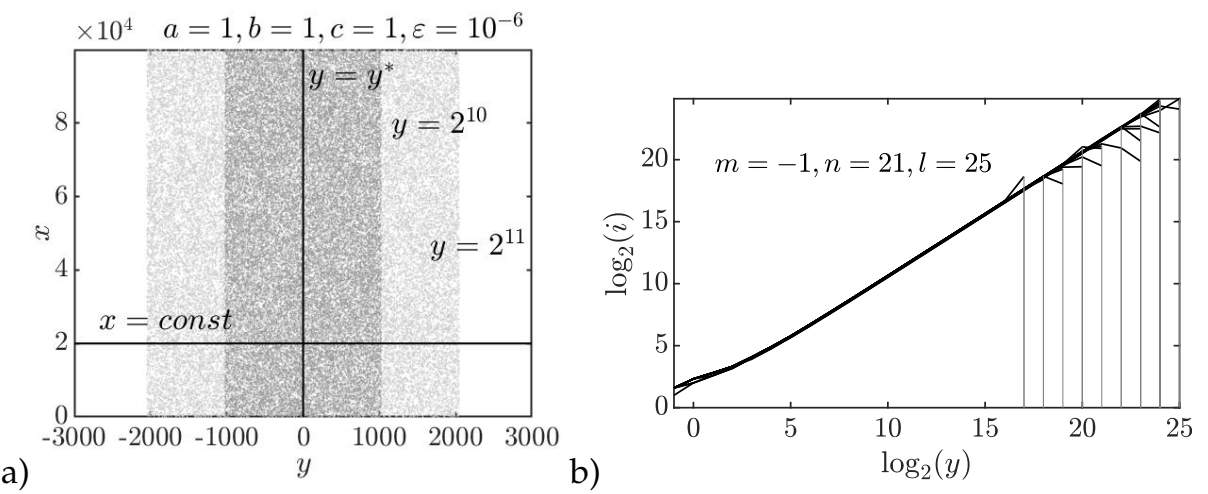

Figure 8. (a) - process of expanding areas of the initial points, (b) - set of curves of the relations between the number of iterations and the width of the basin of attraction.

It occurs that expanding the area by a unit in Figure 8 in a large range of the coordinates $(x, y)$ requires 1.6 iterations on average. Figure $8(\mathrm{a})$ presents the areas of the width of 2048 and 4096 units covered with randomly generated initial points. Figure 8 (b) also illustrates the limitedness of the basin of attraction. It results from achieving 
on every straight line $x=$ const such a value of the coordinate $y=2^{j}$, for which the number of iterations is zero (vertical lines in Figure 8(b)). Lack of convergence of the Newton's method is basically caused by the singularity of the Jacobian matrix $\mathbf{F}^{\prime}(\mathbf{x})$ and, in consequence, inappropriate conditioning of the system (8). The presented construction does not prove the infinity of the attraction set in the direction of the $y$ axis. It proves that it is not a half-plane as it is in the case of the polynomial intersection (15). It may be proved (using MATLAB Symbolic Toolbox) that if $x \rightarrow \infty$ then the eigenvalues of the derivative of projection $\mathbf{G}^{\prime}(\mathbf{x})(26)$ :

$$
\lambda_{x} \rightarrow \frac{-\sqrt{\left(c+\left(-4 a^{2}+8 a b-4 b^{2}+c^{2}\right)\right.}}{2 c}, \lambda_{y} \rightarrow-\frac{\sqrt{\left(c-\left(-4 a^{2}+8 a b-4 b^{2}+c^{2}\right)\right.}}{2 c} .
$$

For the case of the symmetric intersection $a=b=c=1: \lambda_{x}=-1, \lambda_{y}=0$.

The performed calculations indicate that the limit $\lambda_{x}=-1$ is achieved through values almost equal to $\lambda_{x} \cong-1$ but greater than $\lambda_{x}>-1$. Then, the initial points are relatively quickly close to the straight line $y=y^{*}$. However, they slowly converge to $x=x^{*}$. It causes dramatic increase of the number of iterations and the computing time. If $x \rightarrow 0$ and $y \rightarrow \infty$ the Jacobian matrix $\lim _{\substack{x \rightarrow 0 \\ y \rightarrow \infty}} \mathbf{F}^{\prime}(x, y)=\left[\begin{array}{ll}0 & 1 \\ 0 & 1\end{array}\right]$ and its reciprocal $\mathbf{F}^{\prime}(\mathbf{x})^{-1}$ of the considered linear intersection become singular - the Newton's method is not convergent.

In Figure 9 are presented the eigenvalues $\left|\lambda_{\max }\right|$ and the norms of the $\mathbf{G}^{\prime}(\mathbf{x})$ derivative for the linear intersections. Figure 9 also presents the sets of the initial points (lighter
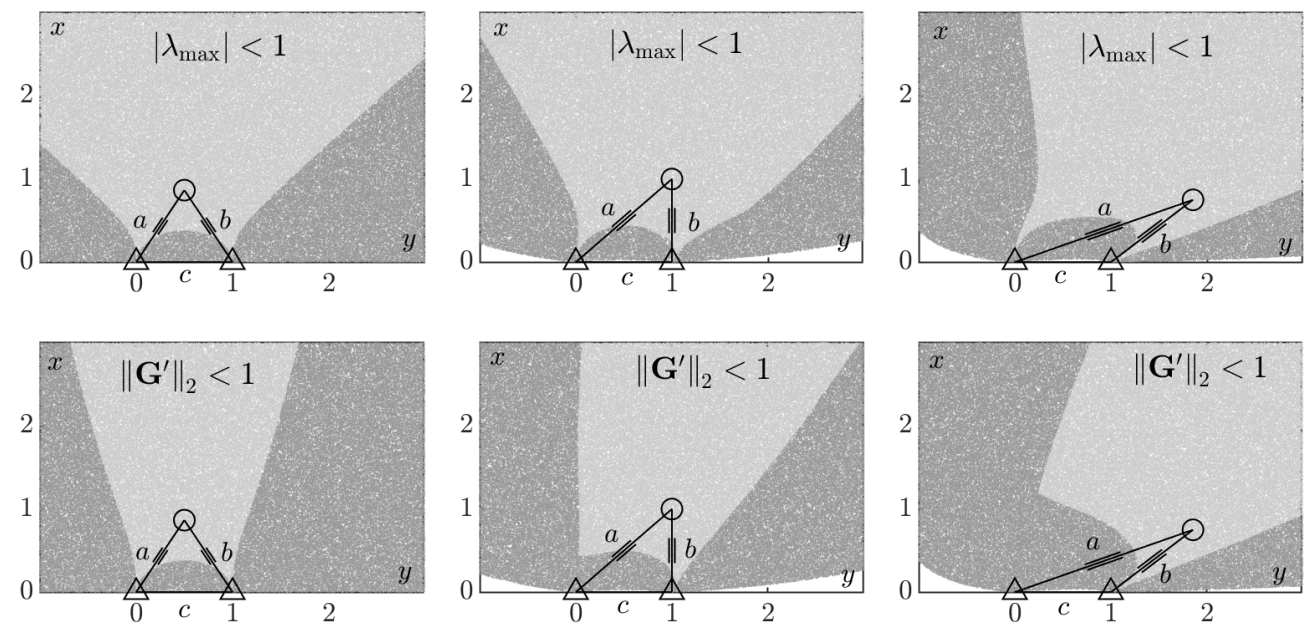

Figure 9. Areas of the initial points $\left|\lambda_{\max }\right|<1,\left\|\mathbf{G}^{\prime}\right\|_{2}<1$ of the linear intersections.

grey areas) for which the maximum eigenvalues $\left|\lambda_{\max }\right|$ and the norms of the derivative $\left\|\mathbf{G}^{\prime}(\mathbf{x})\right\|_{2}$ are smaller than one (generally $\left|\lambda_{\max }\right|<1$ implicates the existence of $\left\|\mathbf{G}^{\prime}(\mathbf{x})\right\|_{2}<1$, see Kincaid and Cheney [6]).

The conducted calculations indicate that the conditions of the above quoted Ostrowski's Theorem 1 and Contractive-Mapping Theorem 2 are satisfied locally in the neighborhood of the solution point. The convergence of the Newton's method for the initial points $\mathbf{x}^{0}$ located far away $\left(\left\|\mathbf{x}^{0}\right\| \gg c\right)$ from the solution point $\mathbf{x}^{*}$, may also be noticed (Figure 9). Furthermore, this figure also indicates that the areas of the initial points $\left\|\mathbf{G}^{\prime}(\mathbf{x})\right\|_{2}<1$ are included in the basins of the linear intersections (25). It means that the condition $\left|\lambda_{\max }\right|<1$, and respectively $\left\|\mathbf{G}^{\prime}(\mathbf{x})\right\|_{2}<1$, is not compulsory for iterative convergence of the Newton's method in the case of the considered intersections. 


\subsection{Schemes of convergence}

Considering the particular case (28) of the contraction condition (Theorem 2), the relations of the distances between the respective initial points in the consecutive iterations of the balls $K\left(\mathbf{x}^{0}\right)$ are studied:

$$
u_{j}=\left\|\mathbf{x}_{j}^{(q)}-\mathbf{x}^{*}\right\| \cdot\left\|\mathbf{x}_{j}^{(s)}-\mathbf{x}^{*}\right\|^{-1}<1, \quad j=1, \ldots, n, \quad q>s=0,1, . .
$$

The satisfying condition (28) means that the initial points $\mathbf{x}^{0}$ successively move closer to the point of the solution $\mathbf{x}^{*}$ of the intersection (Figure 10). The performed simulations indicate that for the intersections presented in Figures 7(b) and (c), inequality (28) is satisfied for the balls $K^{j}\left(\mathbf{x}^{0}\right)$ with odd or even iteration indexes $j$. These balls are separated by the straight line $y=y^{*}$ (Figure 7). Inequality (28) is not satisfied for the subsequent indexes $j$, e.g. $j=0,1$ (Figure $7($ b)) because, in spite of the contraction condition, the area 1 is greater than the area 0 of the initial points. Results equivalent to those presented in Figure 10 were obtained for various random realizations of the sets of the initial points $\mathbf{x}^{0}$.
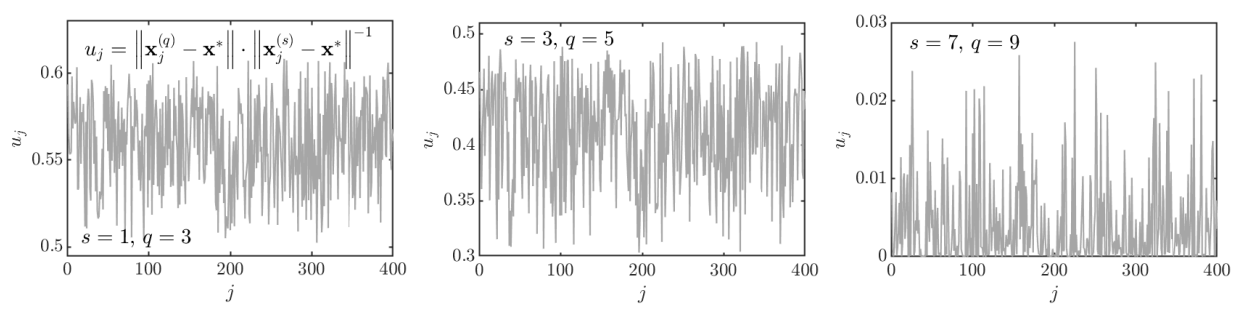

Figure 10. Contraction process of the linear intersection.

According to the Contraction-Mapping Theorem 2, relation (28) for the analyzed cases of the linear intersections may be written as:

$$
\left\|\mathbf{G}^{p}(\mathbf{x})-\mathbf{G}^{p}\left(\mathbf{x}^{*}\right)\right\|=\left\|\mathbf{G}^{k+2}(\mathbf{x})-\mathbf{x}^{*}\right\|<\left\|\mathbf{x}^{k}-\mathbf{x}^{*}\right\|, \forall \mathbf{x} \in K\left(\mathbf{x}^{0}\right), k=0,1, \ldots
$$

The plots in Figure 10 also show that here we have at least linear convergence (12). The contraction coefficients $u_{j}$ decrease from one iteration step to the next, which means increasing speed of the convergence of the Newton's method when point $\mathbf{x}^{*}$ is approached. Generally, the contraction condition of Theorem 2 should be satisfied for every pair $(j, m)$ of points belonging to the ball of the initial points $\left(\mathbf{x}_{j}^{0}, \mathbf{x}_{m}^{0}\right) \in K\left(\mathbf{x}^{0}\right)$, i.e.:

$$
u_{j, m}=\left\|\mathbf{x}_{j}^{(q)}-\mathbf{x}_{m}^{(q)}\right\| \cdot\left\|\mathbf{x}_{j}^{(s)}-\mathbf{x}_{m}^{(s)}\right\|^{-1}<1, j \neq m, j, m=1, \ldots, n, q>s=0,1, \ldots
$$

The conducted calculations indicate that it is not the case because there are subsets of pairs of points belonging to $K\left(\mathbf{x}^{0}\right)$, for which (30) is not satisfied. It is necessary to make an important note that for the balls of the initial points, especially those located appropriately far away from $\mathbf{x}^{0}$, the basic conditions of Theorem 2 are not satisfied because $\mathbf{x}^{*} \notin K\left(\mathbf{x}^{0}\right)$ and $\mathbf{G}(\mathbf{x})$ does not map a closed set $K\left(\mathbf{x}^{0}\right)$ into itself (Figure 7 ). In the case of asymmetric intersections $(a \neq b)$, the situation is more complex and requires additional study - which is beyond the scope of this paper.

\subsection{Basins of attraction of asymmetric linear intersections}

The selected fragments of the asymmetric linear intersections basins of attraction are presented in Figure 11. They are defined by the nonlinear system (16) for various values of the lengths of the distances $a$ and $b$ and the constant (unit) length of the base: $c=1$. They are obtained by covering the selected areas of the function $\mathbf{F}(\mathbf{x})$ (16) domain with grids of various spatial resolutions, which nodes were the initial points of the iterative 
a)

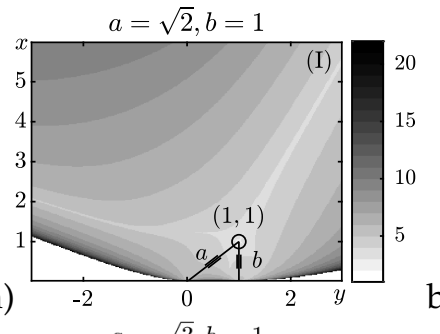

d)
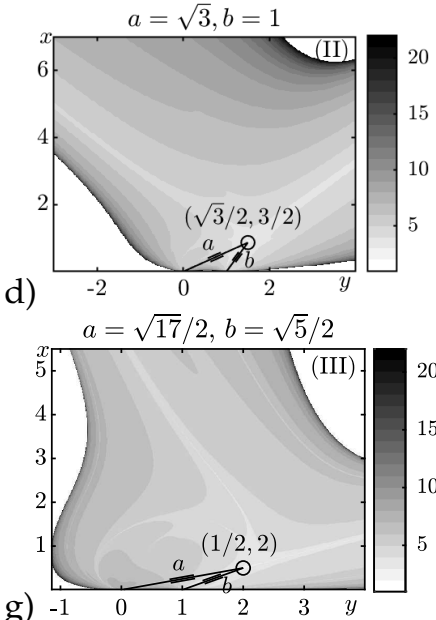

b)
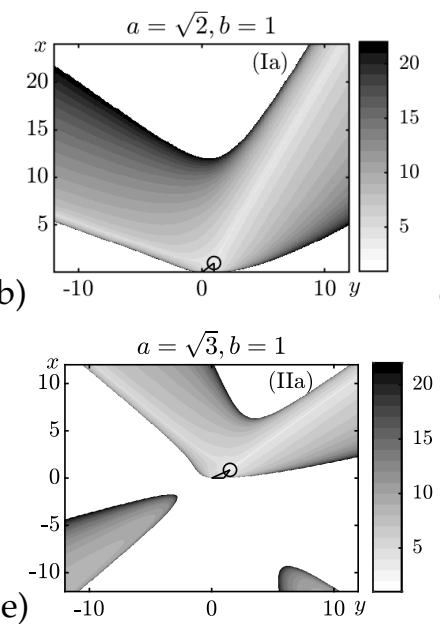

e)

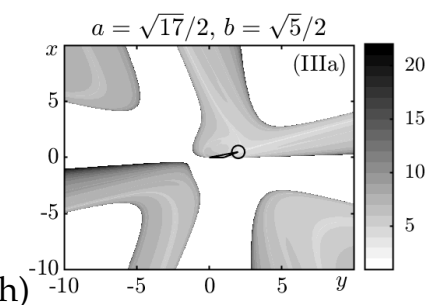

c)
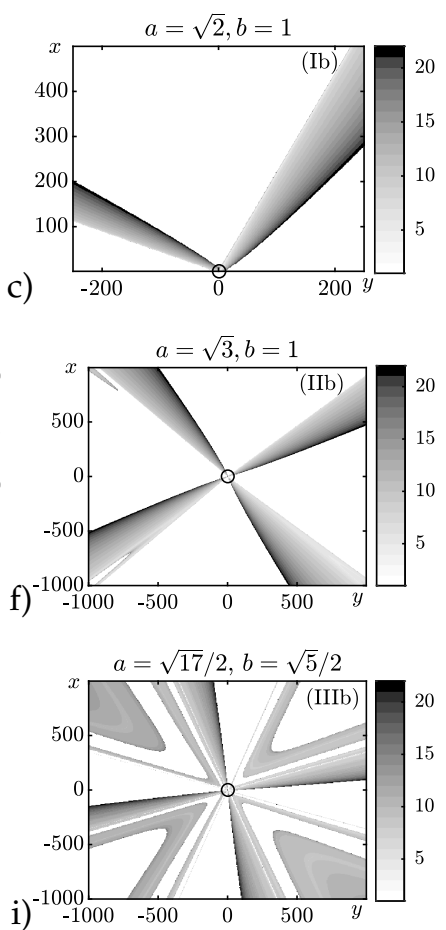

Figure 11. Structures of the basins of attraction of selected asymmetric linear intersections.

Newton's method. Unlike the basins of linear symmetric intersections, the asymmetric basins have more complex geometric and iterative structure. The differences grow with the increase of the departure from symmetry (see Figure 11). It may be noticed in Figure 11 that the structures of these basins depend on the intersection shape determined by the values of measurements $a$ and $b$. In general, they are disconnected and usually composed of numerous separate subsets, which we call "branches". The branch that contains the intersection is regarded as the main one. The performed calculations indicate that the branches are observable in a wide range of coordinates values: $x= \pm 10^{17}, y= \pm 10^{16}$ expressed in the units defined by the length of the intersection base $c$ (see Figure 12).

The plots in Figure 12 are obtained by increasing the areas (see Figures 11(g), (h), (i)) to which the randomly generated initial points belonged.
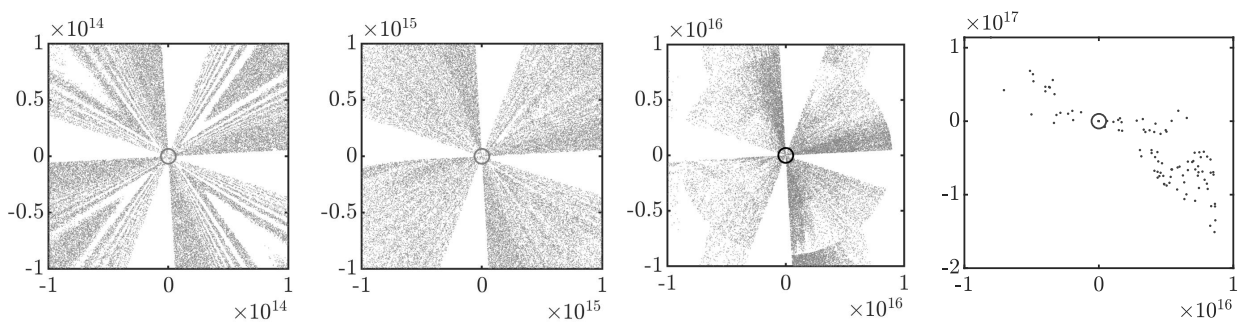

Figure 12. Fragments of the basin of attraction of the linear intersection in a wide range of coordinates values.

In the case of the last plot in Figure 12, the area of: $\left\{x= \pm 10^{18}, y= \pm 10^{18}\right\}$ was declared. Analyses of the results of numerous realizations of the generated points show that the convergent ones belong to a smaller area: $\left\{x= \pm 10^{17}, y= \pm 10^{16}\right\}$. It may therefore be believed that the basin of attraction in this case is finite. When we apply the analytical approach to the study of the behavior of the Jacobian matrix $\mathbf{F}^{\prime}(\mathbf{x})$ of system (16) at the limit $(x \rightarrow \infty, y \rightarrow \infty)$, in the straight lines bunch: $x-x^{*}=\alpha\left(y-y^{*}\right)$ passing through point $\mathbf{x}^{*}$ of the intersection solution it occurs that the limit Jacobian matrices 
$\lim _{\substack{x=\alpha\left(y-y^{*}\right)+x^{*} \rightarrow \infty \\ y \rightarrow \infty}} \mathbf{F}^{\prime}(x, y)=\frac{1}{\sqrt{\alpha^{2}+1}}\left[\begin{array}{cc}\alpha & 1 \\ \alpha & 1\end{array}\right]$ are singular for every straight line of the bunch. It indicates that for very large values of the coordinates $(x, y)$ of the initial points, the Newton's method is not convergent. The practice shows that the convergence is lost for finite values of the order of $\pm 10^{17}$.

Studying the impact of the limit number of iterations on the basins of attraction of the linear intersection, we found that increasing the number may impact their geometric structure. Figures 13(a) and (b) show the change of the width of the intersection basin branch (Figure 11(f)) with the increase of the limit value from 20 to 1000 iterations. Figures 13(c) and (d) illustrate the differences between the basins obtained for 1000 and

a)

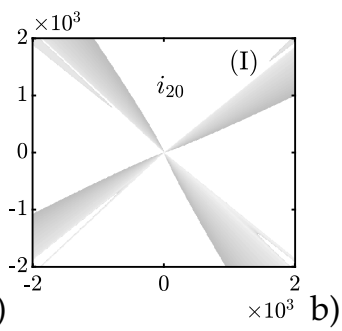

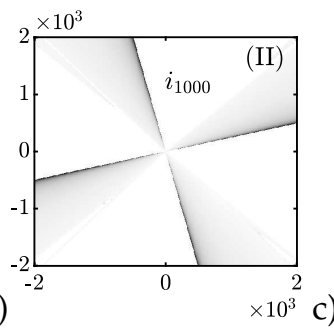

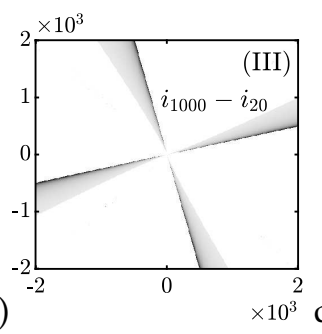

d)

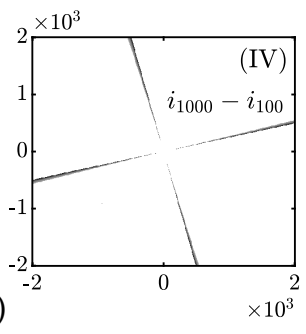

Figure 13. The impact of the number of iterations on the geometric structure of the basin of attraction.

20 iterations, and for 1000 and 100 iterations. In the latter case it is a trace difference. It means a certain state of saturation, i.e. for large values of the number of iterations, the process of widening is insignificant. Then, the shape and the area of the basins of attraction are practically unchanged.

Analyzing the cross-sections (Figure 14), it is found that there are boundary initial points $\mathbf{x}^{0}$ which very slowly converge to the solution $\mathbf{x}^{*}$. It is determined by the initial phase of the iteration characterized by linear convergence with the value of parameter $\alpha \approx 1$ (12). When point $\mathbf{x}^{*}$ is being approached, the speed of convergence increases becoming quadratic (11) in an appropriately small neighborhood of $\mathbf{x}^{*}$. The crosssections show that the slowly convergent boundary initial points $\mathbf{x}^{0}$ are concentrated on the edges of the branches of the basin of attraction. It can also be seen in Figures 11 to 16.

a)
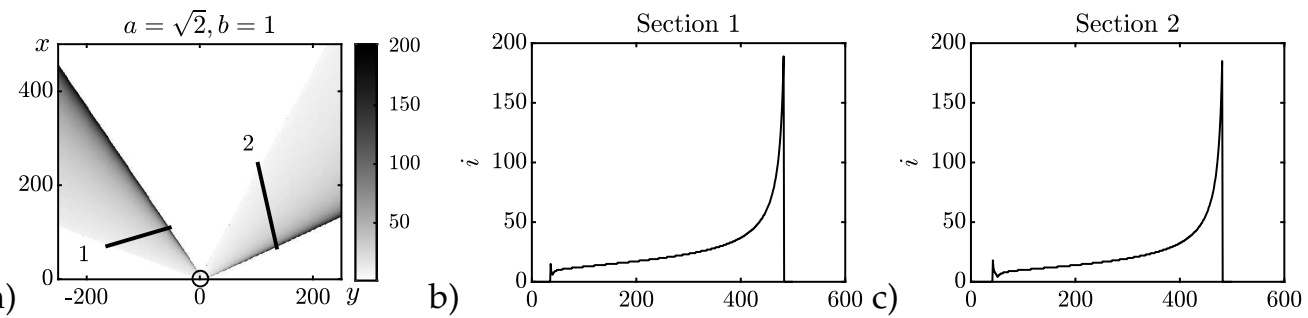

Figure 14. The number of iterations in the cross-sections of the basin of attraction.

Figure 15 presents the basins of asymmetric linear intersections of the family $b=$ $c=1,1 \leq a<2$ with the increasing value of the distance $a$. It may be noticed that with the continuous change of the length of the distance $a$, which is also the case for other families, the geometric structure of the basins of attraction changes qualitatively. Such qualitative changes of the basin structure have therefore the features of bifurcation phenomena observed in dynamic systems (Guckenheimer and Holmes [4]).

The plots in Figure 16 present the fragments of the basins of attraction of asymmetric intersection. They are the result of successive (ten-time) increase of the size of the grid of the initial points. It may be noticed that in the subsequent figures similar geometric structures appear as in the previous figures. These observed earlier in better resolution (for smaller grid distance), here in the lower resolution appear as sets of lines (see e.g. 

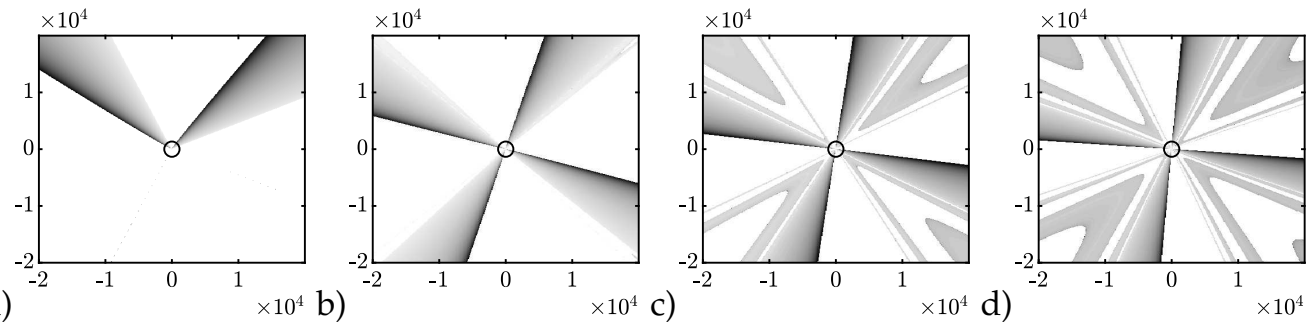

Figure 15. Bifurcation of the family of the linear intersection - the number of iterations: 500, $\varepsilon=10^{-6}$.

the last plot in Figure 16). In general, it may be concluded that the basins of attraction of linear intersections which are strongly asymmetric, look similar in larger scale and in smaller scale. This remark also concerns their iterative structures. Such invariance is characteristic of self-similar structures (Barnsley [13]).
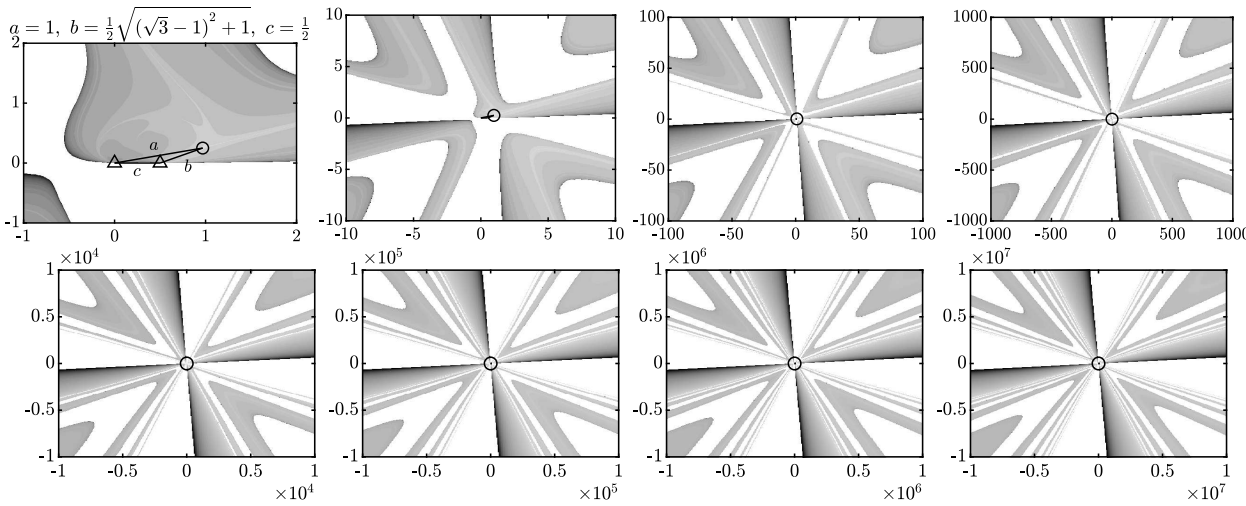

Figure 16. Self-similarity of the geometric and iterative structures of the basin of attraction.

\section{Angular intersection}

The angular intersection is a task of determining coordinates of the intersected point $C$ using measured angles $\alpha$ at point $A$ and $\beta$ at point $B$ (Figure 17). In order to simplify the equations used in the paper it is assumed (without any loss of generality) that the intersection base of the length of $c$ coincides with the $y$ axis of the geodetic coordinate frame, i.e.: $x_{A}=x_{B}=0, y_{A}=c, y_{B}=0$.

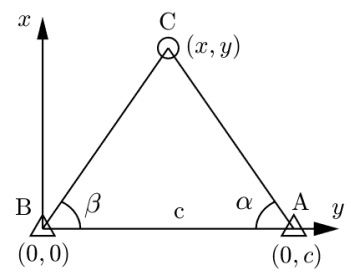

Figure 17. Angular intersection.

In this frame, the analytic solution may be obtained e.g. from the following relation (Ghilani [3]; Uren and Price [11]):

$$
\left\{x=\frac{c}{\cot (\alpha)+\cot (\beta)}, \quad y=\frac{c \cdot \cot (\beta)}{\cot (\alpha)+\cot (\beta)} .\right.
$$

\subsection{Basins of attraction of angular intersection}

For the study of the properties of basins of attraction of the angular intersection, we construct a non-linear system of observation equations, binding angular measurements 
$\alpha$ and $\beta$ with the coordinates of the intersected point $(x, y)$ and the coordinates of the base $c$ :

$$
\begin{aligned}
& \mathbf{F}_{1}(x, y)=\left\{\begin{array}{l}
f_{1}(x, y)=\frac{\pi}{2}+\arctan \frac{y-c}{x}-\alpha=0 \\
f_{2}(x, y)=\frac{\pi}{2}-\arctan \frac{y}{x}-\beta=0
\end{array}, x>0\right. \\
& \mathbf{F}_{2}(x, y)=\left\{\begin{array}{l}
f_{1}(x, y)=\frac{3 \pi}{2}-\arctan \frac{y-c}{x}-\alpha=0 \\
f_{2}(x, y)=\frac{\pi}{2}+\arctan \frac{y}{x}-\beta=0
\end{array}, \quad x<0\right.
\end{aligned}
$$

This form of the system is considering the possibility to assume any point belonging to the plane, i.e. $\mathbf{x}^{0} \in D \subset \mathbb{R}^{2}$ for an initial point $\mathbf{x}^{0}$ of the Newton's iterative procedure. Equations (32) express the differences of the angles calculated using the iterations of the coordinates $\mathbf{x}$ of the initial point $\mathbf{x}^{0}$ and the measured angles: $\alpha$ and $\beta$. The angles determined using the coordinates are the differences of azimuths of the left and right angle arms respectively for $x>0$ and $x<0$. Relations in (32) are true for the assumed location of the intersection base (Figure 17). It may be noticed that systems (32) is invariant with respect to translation and isotropic scaling (Helmert transformation), i.e. replacement of coordinates: $x^{\prime}=t_{x}+s \cdot x, y^{\prime}=t_{y}+s \cdot y$. Because of this the properties of the basins of attraction of the transformed systems are the same. Considering (32) the following iterative system may be written (as a fixed-point systems):

$$
\mathbf{x}=\mathbf{G}(\mathbf{x}), \quad \mathbf{G}(\mathbf{x})= \begin{cases}\mathbf{x}-\mathbf{F}_{1}^{\prime}(\mathbf{x})^{-1} \mathbf{F}_{1}(\mathbf{x}), & x>0 \\ \mathbf{x}-\mathbf{F}_{2}^{\prime}(\mathbf{x})^{-1} \mathbf{F}_{2}(\mathbf{x}), & x<0\end{cases}
$$

Relation (32) indicates that for the Jacobian matrix $\mathbf{F}^{\prime}(\mathbf{x})$ and its reciprocal $\mathbf{F}^{\prime}(\mathbf{x})^{-1}$ the following relations are satisfied:

$$
\mathbf{F}_{2}^{\prime}(\mathbf{x})=-\mathbf{F}_{1}^{\prime}(\mathbf{x}), \quad \mathbf{F}_{2}^{\prime}(\mathbf{x})^{-1}=-\mathbf{F}_{1}^{\prime}(\mathbf{x})^{-1}, \quad \mathbf{F}_{1}^{\prime}(\mathbf{x})=\left[\begin{array}{cc}
\frac{c-y}{x^{2}+(c-y)^{2}} & \frac{x}{x^{2}+(c-y)^{2}} \\
\frac{y}{x^{2}+y^{2}} & \frac{-x}{x^{2}+y^{2}}
\end{array}\right]
$$

It may be noticed that $\mathbf{F}^{\prime}(\mathbf{x})$ and $\mathbf{F}^{\prime}(\mathbf{x})^{-1}$ are singular on the straight line $x=0$, in particular in the points determining the base $(x=0, y=0)$ and $(x=0, y=c)$.

\subsection{Area of convergence of the Newton's method of angular intersection}

The basins of attraction of the angular intersection for various measured values of angles $\alpha, \beta$, assumed values of accuracy $\varepsilon_{x}=\varepsilon_{F}=10^{-6}$ and the maximum number of iterations $i_{\max }=10^{3}$ are presented in Figure 18. Analyzing the obtained results, it can be noticed that the basins of symmetric intersections (Figures 18(a), (b) and (c)) are symmetric. The straight line perpendicular to the line segment of the intersection base passing through the solution point is the axis of symmetry. The basins of mirror intersections (Figures 18(a), (b)) have no shared parts. It proves (indirectly) the appropriate defining of the observation equation set in (32) and the iterative function (33). The existence of such parts would mean ambiguity, i.e. the existence of the initial points simultaneously convergent to the solutions of different intersections

The areas of the convergent initial points presented in Figure 18 are limited. Their linear sizes correspond to a few units of the intersection base. In order to verify this observation a series of tests concerning covering the intersections by grids of large sizes $x^{0}, y^{0} \in\left(-10^{6}, 10^{6}\right)$ or by randomly generated sets of the initial points (13) are conducted. The maximum number of iterations necessary to achieve the solution $\mathbf{x}^{0}$ is also studied. For the assumed accuracy $\varepsilon_{x}=\varepsilon_{F}=10^{-6}$ it did not exceed 23 (gray-scale bar legend in Figure 18). The iterative structures presented in Figure 18 corresponding to various intersections are similar. The basins of asymmetric intersections (Figures 
a)
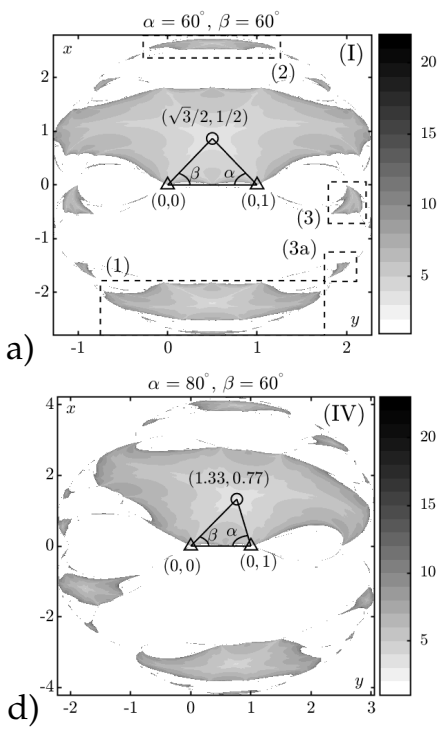

b)
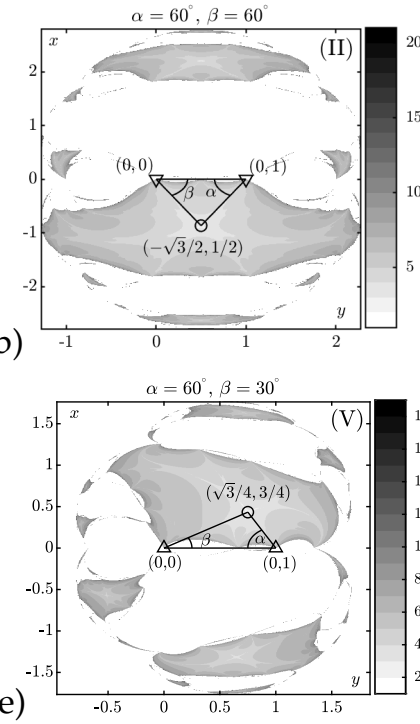

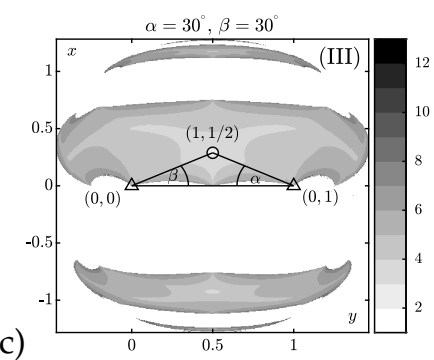

c)

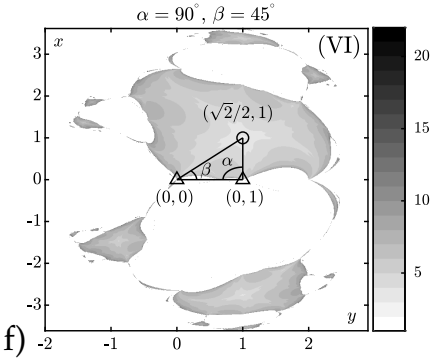

Figure 18. Basins of attraction of angular intersections.

18(d), (e), and (f)) are deformed versions of the symmetric intersections (Figure 18(a)), i.e. they are homeomorphic. This observation does not apply to the basin shown in Figure 18(c) because it lacks e.g. subsets of the type: Figures 19(d), (e), visible in Figure 18(a). Such subsets are also missing in the basins of intersections for angles $\alpha=\beta<40^{\circ}$ (the limit values of the angles for which the structures of the type presented in Figures 19(d) and (e) disappear are not studied in details). Therefore, it may not be stated that in every case a continuous change of angles $\alpha, \beta$ is followed by a continuous change of the geometric structures. The main area including the intersection and subordinate areas may be distinguished in the case of each of the basins. They may be seen especially in the magnifications of the rectangular areas: Figures 19(a) to (d), marked in Figure 18(a) as (1), (1a), (2), (3), and (3a). Magnification (1) in Figure 19 reveals the geometric similarity and similarity of the iterations distribution with the main area. This similarity may be noticed (Figure 19) for area (1a), then (1b) etc. It seems that fragments (3) and (3a) in Figure 19 have a different geometric and iterative structure than the main area of the basin of attraction. However, from the topological point of view, they are probably homeomorphic. Increasing in this case the resolutions of the grid of the initial points, one may also find the fragments similar to it ((3a) in Figure 19). Such behavior is characteristic of self-similar sets (Barnsley in [13]).

a)

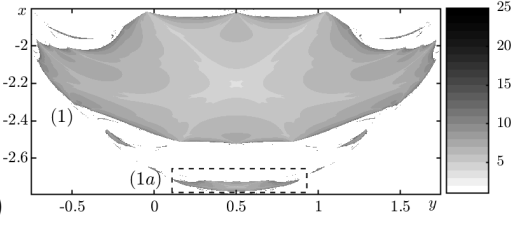

b)

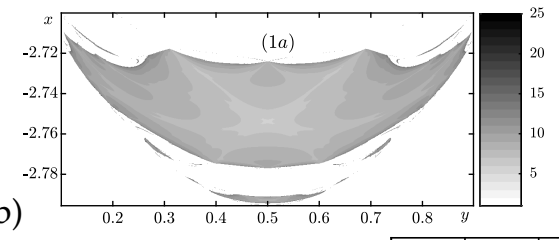

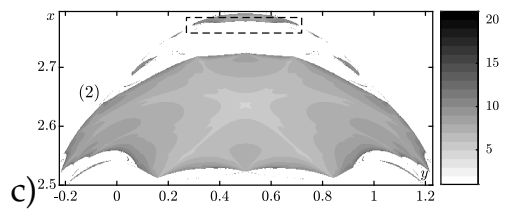
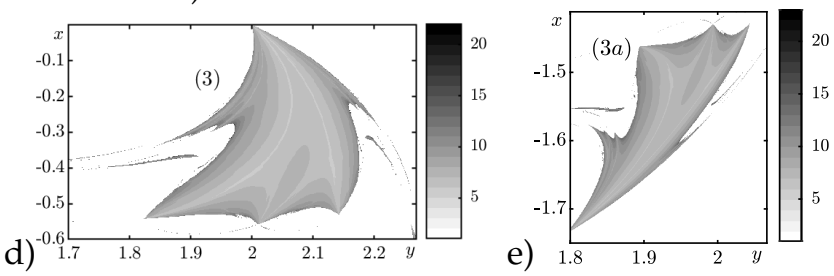

Figure 19. Magnified fragments of the basin of attraction of symmetric angular intersection.

The conducted analysis indicates that basins of attraction of the angular intersections are spatially limited and disconnected sets. They are hypothetically composed of 
an infinite number: $B=\bigcup_{j=1}^{\infty} B_{j}$ of the subsets $B_{j}$ of the initial points, which demonstrate features of self-similarity. Generally, the initial points $\mathbf{x}^{0}$ of the deeper embedded sets (of greater values of the index $j$ ) converge more slowly, but - as it is indicated by the results presented in Figures 19 and 20 - the number of iterations may be comparable with the number of iterations of the main structure. For this reason, the following question was asked: how do the initial points $\mathbf{x}^{0}$ belonging to various fragments of the basin of attraction (see Figure 20) converge?
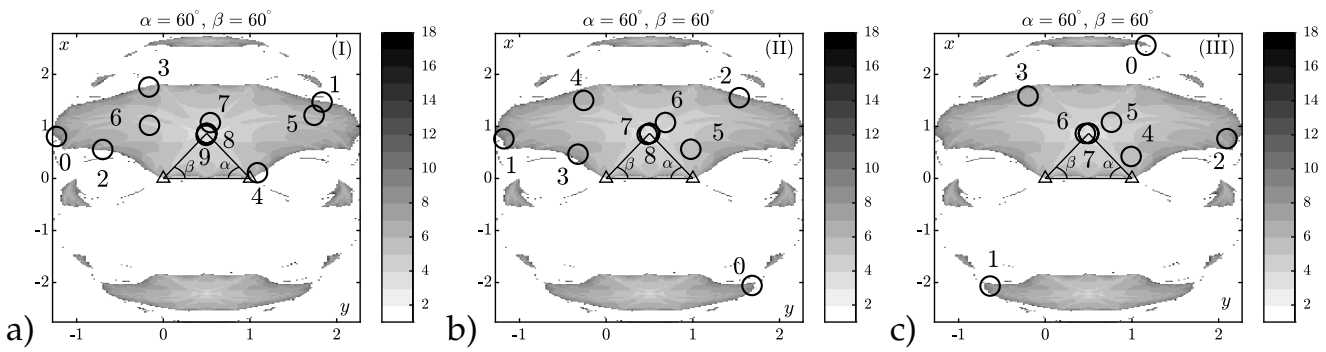

Figure 20. Convergence of the initial points for various fragments of the basin of attraction of the angular intersection.

If the initial point (denoted by 0 ) is within a fragment of the main basin, then the points of the following iterations converging to the solution remain within that fragment. The number of iterations depends on the location of the start - the light areas correspond to the smaller number of iterations. In the case when it is started from a fragment of Figure 20(b), there is a snap through to the main fragment of similar number of iterations. Analogically, the initial points (Figure 20(c)) firstly snap through to the bottom fragment, and then to the main fragment after which they converge, depending on the location in the fragment, to the intersection solution in a few iterations. The described mechanism causes that the initial points $\mathbf{x}^{0} \in B_{j}$ located in the areas of small numbers of iterations converge very quickly (in this case, it may be considered as a specific "tunneling" phenomenon). Abstractly thinking, an infinite series of iterations corresponding to an infinite series of subsets $B_{j}(j=1, \ldots \infty)$ belonging to the families of the initial points of the basin of attraction may theoretically be considered. Then the number of iterations necessary to achieve the solution $\mathbf{x}^{*}$ is infinite. In practice it means that there is a possibility to assume for $\mathbf{x}^{0}$ a point which is convergent after realizing a very large number of iterations. The probability of choosing such a point $\mathbf{x}^{0} \in B_{j}$ is, however, relatively small. It decreases with increasing index $j$ because the areas occupied by the sets $B_{j}$ decrease very quickly.

The eigenvalues $\left|\lambda_{\max }\right|$ and norms of the derivative $\mathbf{G}^{\prime}(\mathbf{x})$ of the projection $\mathbf{G}(\mathbf{x})$ of angular intersection are also studied. Due to a high complexity of the relations defining them, they are not in the paper in their full form. Figure 21 presents the sets of the initial points $\mathbf{x}^{0}$ (darker grey areas) for which the norm $\left\|\mathbf{G}^{\prime}(\mathbf{x})\right\|_{2}$ is smaller than one: $\left\|G^{\prime}\left(\mathbf{x}^{0}\right)\right\|_{2}<1$. For the considered intersections (Figure 21), all points of the sets from $\left\|\mathbf{G}^{\prime}\left(\mathbf{x}^{0}\right)\right\|_{2}<1$ converge to the solutions $\mathbf{x}^{*}$ because they are entirely contained in the basins of attraction of the intersections.

They are not local sets such as result from the differentiability of $\mathbf{G}(\mathbf{x})$ in the solution point $\mathbf{x}^{*}$ and are usually taken into account in theorems concerning convergence of the Newton's method (see e.g. Ostrowski's Theorem 1). Inequality of the areas of the basins and sets with $\left\|\mathbf{G}^{\prime}\left(\mathbf{x}^{0}\right)\right\|_{2}<1$ indicates that there are areas of basins with $\left\|\mathbf{G}^{\prime}\left(\mathbf{x}^{0}\right)\right\|_{2}<1$ which confirms that the condition provided in Ostrowski's Theorem 1 is not a necessary condition for convergence [7]. Theoretically, existence of such areas of $\left\|\mathbf{G}^{\prime}\left(\mathbf{x}^{0}\right)\right\|_{2}<1$ may result from the Newton-Kantorovich Theorem 2 which makes no assumptions about the existence of a solution. It shows that if $\mathbf{F}^{\prime}\left(\mathbf{x}^{0}\right)$ is non-singular and Lipschitz continuous in a region containing $\mathbf{x}^{0}$, which is satisfied for the considered intersections, and the first step of the Newton's method is sufficiently small, relative to 

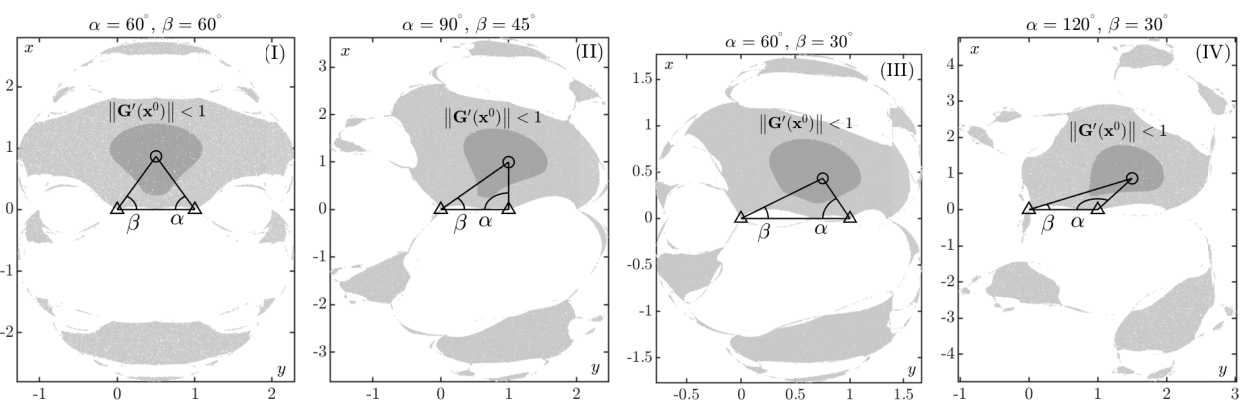

Figure 21. Areas of the iterative Newton's method initial points for $\left\|\mathbf{G}^{\prime}(\mathbf{x})\right\|_{2}<1$.

the nonlinearity of $\mathbf{F}(\mathbf{x})$ defined by Dennis and Schnabel in [2], then there must be a root in this region. It is necessary to note that the indicated theorems do not apply in the case of the disconnected basins of attraction.

\section{Combined linear intersection and angular resection}

The combined linear intersection and angular resection (see Figure 22) is a task of performing two inhomogeneous observations in the $\mathrm{ABC}$ triangle: the angular of the angle $\gamma$ at point $C$ and the linear one presented as the length of the straight line segment $|\mathrm{BC}|=a$. The calculation of the coordinates of the points determined by means of the combined linear intersection and angular resection, may come down to solving the angular intersection using the sine theorem $(c \sin \alpha=a \sin \gamma)$ for calculating angles: $\alpha$ and $\beta=180^{\circ}-(\alpha+\gamma)$.

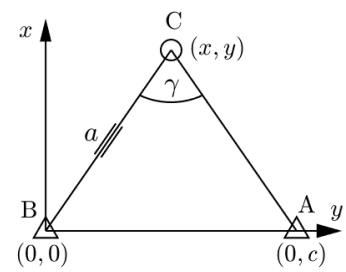

Figure 22. Combined linear intersection and angular resection.

\subsection{Basins of attraction of the combined intersection and resection}

In order to simplify the equations, the intersection base is assumed to be of a unit length $(c=1)$ and coinciding with the $y$ axis of the geodetic coordinate frame, i.e.: $x_{A}=x_{B}=0, y_{A}=c=1, y_{B}=0$. In this frame, a non-linear system of observation equations binding the measurements of $a, \gamma$ and the coordinates of the intersected point $(x, y)$ has the form of:

$$
\begin{aligned}
& \mathbf{F}_{1}(x, y)=\left\{\begin{array}{l}
f_{1}(x, y)=\sqrt{x^{2}+y^{2}}-a=0 \\
f_{2}(x, y)=\arctan \frac{y}{x}-\arctan \frac{y-c}{x}-\gamma=0
\end{array}, \quad x>0\right. \\
& \mathbf{F}_{2}(x, y)=\left\{\begin{array}{l}
f_{1}(x, y)=\sqrt{x^{2}+y^{2}}-a=0 \\
f_{2}(x, y)=\arctan \frac{y-c}{x}-\arctan \frac{y}{x}-\gamma=0
\end{array}, \quad x<0\right.
\end{aligned}
$$

The systems in (35) are written in the iterative form (the superscripts are omitted):

$$
\mathbf{x}=\mathbf{G}(\mathbf{x}), \quad \mathbf{G}(\mathbf{x})= \begin{cases}\mathbf{x}-\mathbf{F}_{1}^{\prime}(\mathbf{x})^{-1} \mathbf{F}_{1}(\mathbf{x}), & x>0 \\ \mathbf{x}-\mathbf{F}_{2}^{\prime}(\mathbf{x})^{-1} \mathbf{F}_{2}(\mathbf{x}), & x<0\end{cases}
$$

where after introducing the following denotations: 
$d=x / \sqrt{x^{2}+y^{2}}, e=y / \sqrt{x^{2}+y^{2}}, g=(c-y) / \sqrt{x^{2}+(c-y)^{2}}, h=x / \sqrt{x^{2}+(c-y)^{2}}$,

the Jacobian matrices have the form:

$$
\mathbf{F}_{1}^{\prime}(x, y)=\left[\begin{array}{cc}
d & e \\
-(g+e) & (d-h)
\end{array}\right], \quad \mathbf{F}_{2}^{\prime}(x, y)=\left[\begin{array}{cc}
d & e \\
(g+e) & (d-h)
\end{array}\right]
$$

Equations 35 are invariant with respect to translation and isotropic scaling of coordinates: $x^{\prime}=t_{x}+s \cdot x, y^{\prime}=t_{y}+s \cdot y$ and measurements: $a^{\prime}=s \cdot a, b^{\prime}=s \cdot b$ and $c^{\prime}=s \cdot c$. Jacobian matrices $\mathbf{F}_{1}^{\prime}$ and $\mathbf{F}_{2}^{\prime}$ are singular for points on the straight line $x=0$. Figure 23 presents basins of attraction of the combined intersection and resection for the assumed (unit) length of the base and selected lengths of the distance $a$ and angle $\gamma$.

a)

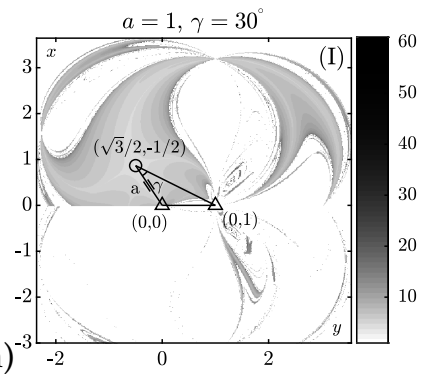

d)
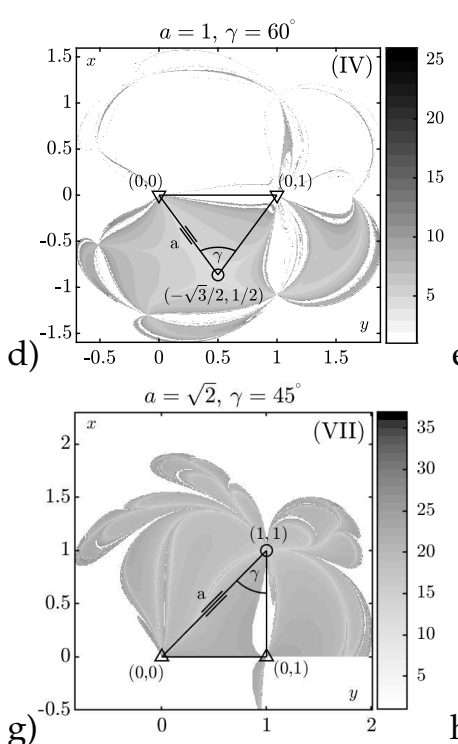

b)
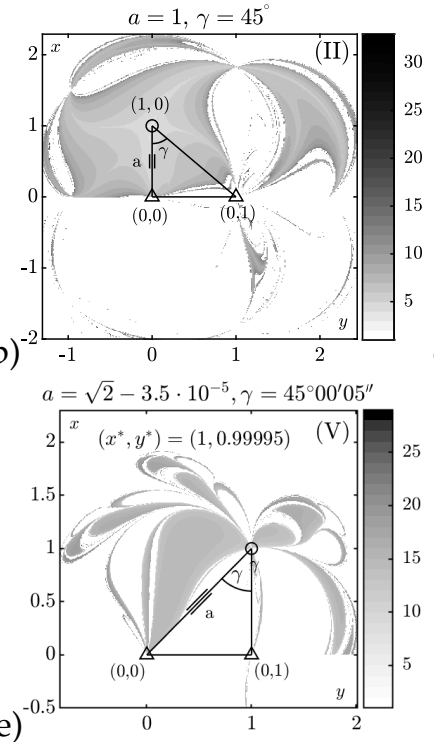

e)

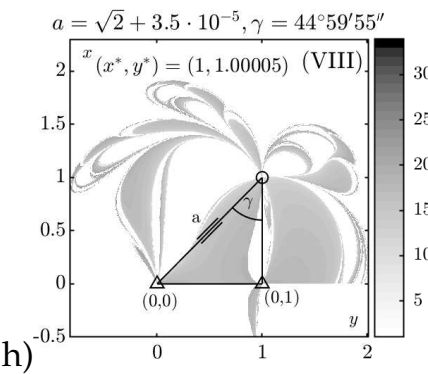

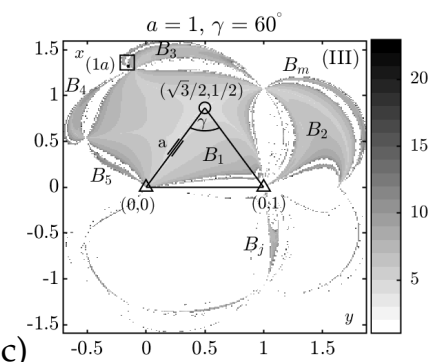

C)
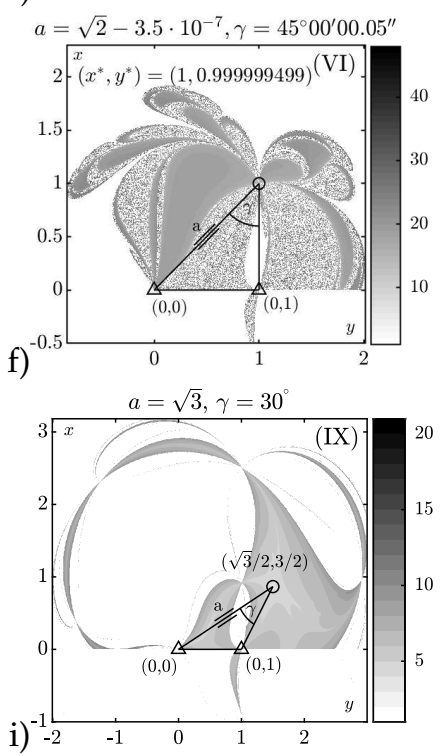

Figure 23. Basins of attraction of the Newton's iterative method of the combined intersection and resection.

They belong to the family of combined intersections and resections defined by the inequalities: $c=1,0<a<2, b<a+c$. Figures 23(c) and (d) present mirror combined intersections and resections $\left(a=1, \gamma=60^{\circ}\right)$. Their basins are separate, which (according to the theory) indirectly confirms correctness of the equation systems in (35). It may be noticed that the geometric and iterative structures of the basins (for the established: limit number of iterations $-i_{\max }$ and accuracy $\varepsilon_{x}, \varepsilon_{F}$ ) are homeomorphic, i.e. they change continuously with continuous change of measurements: $a$ and $\gamma$. Basins corresponding to close neighborhood of $\gamma=45^{\circ}$ (Figures 23(e) to (h)) are an exception. It can be seen (Figure 23(g)) that the set is composed of sets of the type (Figures 23(e) and (h)). Figure 23(f) also presents one of the transitional forms very close to the basin 
of $\gamma=45^{\circ}$. In the "dotted" areas of the basin there are both convergent and divergent initial points. Generally, decreasing or increasing the value of angle $\gamma, \gamma \neq 45^{\circ}$ causes switching the geometric structures of the basins between the types (Figures 23(e) and (h)) in both ways. Such behavior results from the jumping (Figure 24) character of the function course $f_{2}(x, y)$ of the observation system in (35).

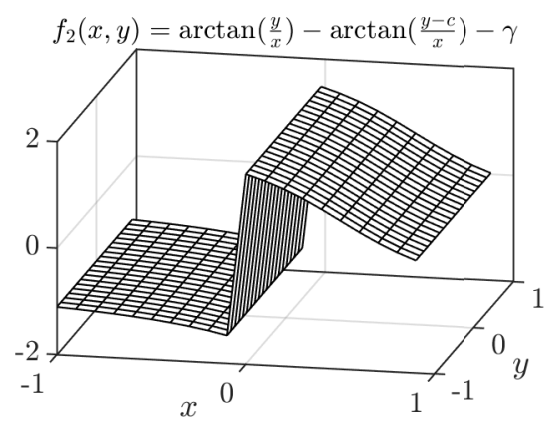

Figure 24. Jumping course of function $f_{2}(x, y), c=1$.

It was also noticed that for $\gamma$ approaching $45^{\circ}$ results in a quick decrease of the neighborhood radius of the intersected point determined by the intersected point and its closest divergent point (see Figures 25(a) and (c)). Although also in these cases the areas of basins are appropriately large, there may be divergent points in a very small neighborhood of the intersected point. This effect is disadvantageous from the practical computational point of view. In neither of the analyzed cases (Figure 23) the radiuses did exceed the unit value of the base length.
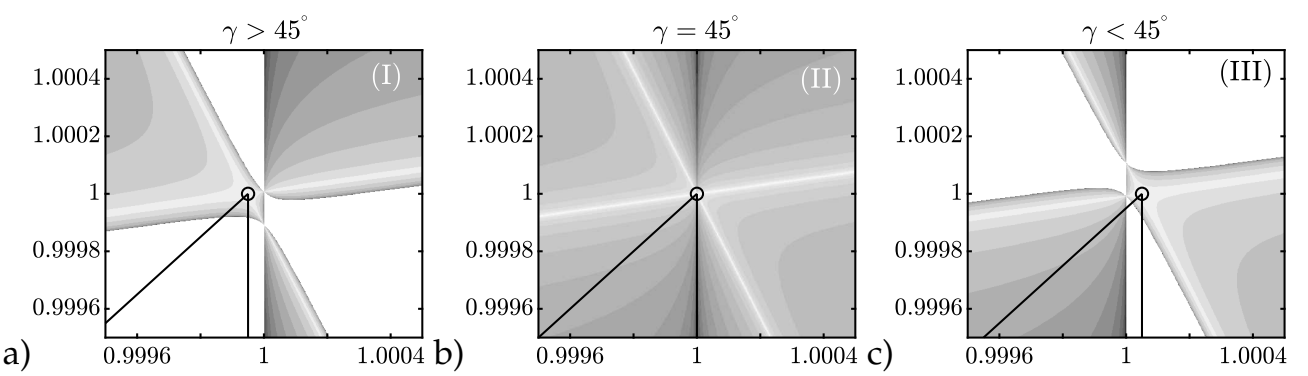

Figure 25. Neighborhoods of the solution points for $\gamma \geq 45^{\circ}$ and $\gamma<45^{\circ}$.

Studying geometric structures of combined intersections and resections shows that similar to angular intersections - they are limited and disconnected, i.e. composed (theoretically) of an infinite: $B=\bigcup_{j=1}^{\infty} B_{j} ; j$ - number of subsets $B_{j}$ of convergent initial points (Figures 23 and 26). It is the result of analyses conducted for successively increased resolutions of grids of the initial points in Figure 23. The analyses showed that the subsets have similar geometric and iterative structures. In the neighborhood of each of the subsets there are similar subsets which become visible after appropriate increase of resolution (Figure 26). Theoretically, this procedure may be repeated any number of times.
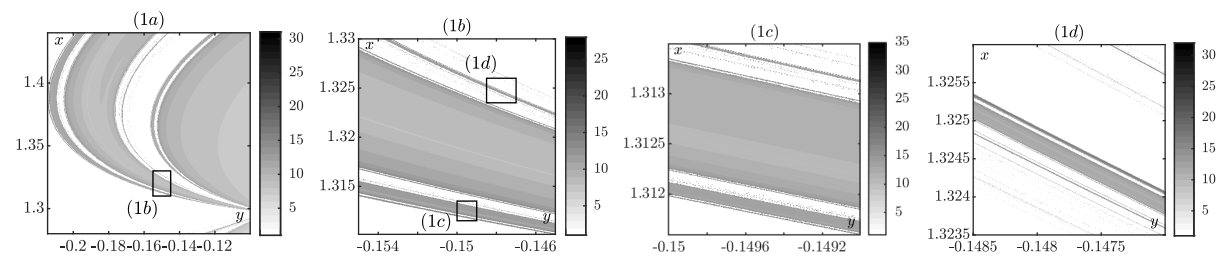

Figure 26. Structure of the basin fragment (1a) (see Figure 23 (c)) for increasing resolution of the starting point grids. 
Similar results as for angular intersections are obtained when we study the process of convergence of the initial points $\mathbf{x}^{0}$ from various fragments of basins of attraction (Figure 27).
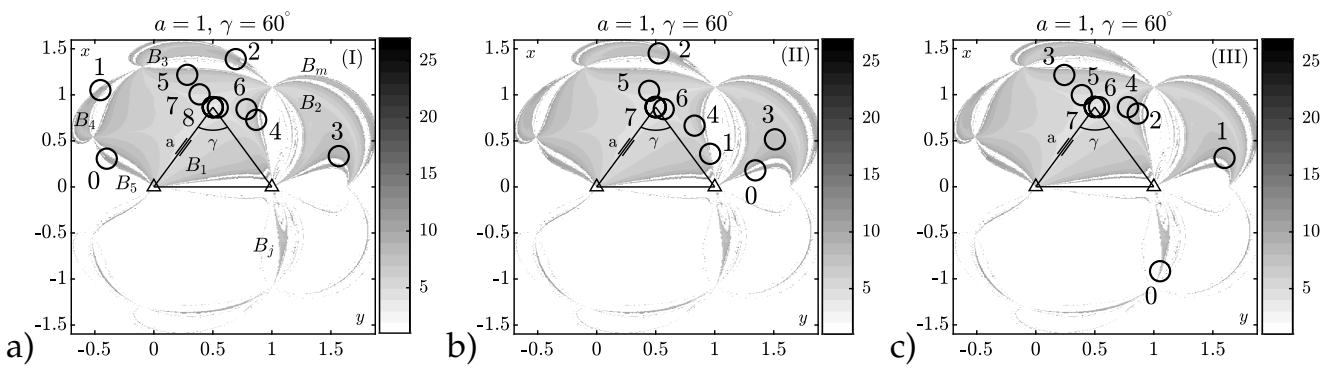

Figure 27. Initial points convergence schemes.

Reaching the main fragment $B_{1}$, which includes the intersected point by an initial point (its iterations) is related with performing a certain number of snaps through $B_{k} \rightarrow B_{j}, k>j$ between the subsets $B_{k}, B_{j}$ of the basin (Figure 27). This number depends on the location of the start and it is usually greater than the number of iterations of angular intersections. It is characteristic that only one iterative point corresponds to subset $B_{j}, j=1, \ldots, n$. The conducted analysis indicates that a greater number of iterations is possible only within the main fragment of the basin. Like for angular intersections, the snaps through occur into areas of similar number of iterations. We notice that for the initial points belonging to different sets $B_{j}$ there are various schemes of convergence (Figures 27(a) and (c)). It may signify that sets $B_{j}$ belong to different families, classifiable on the basis of the schemes.
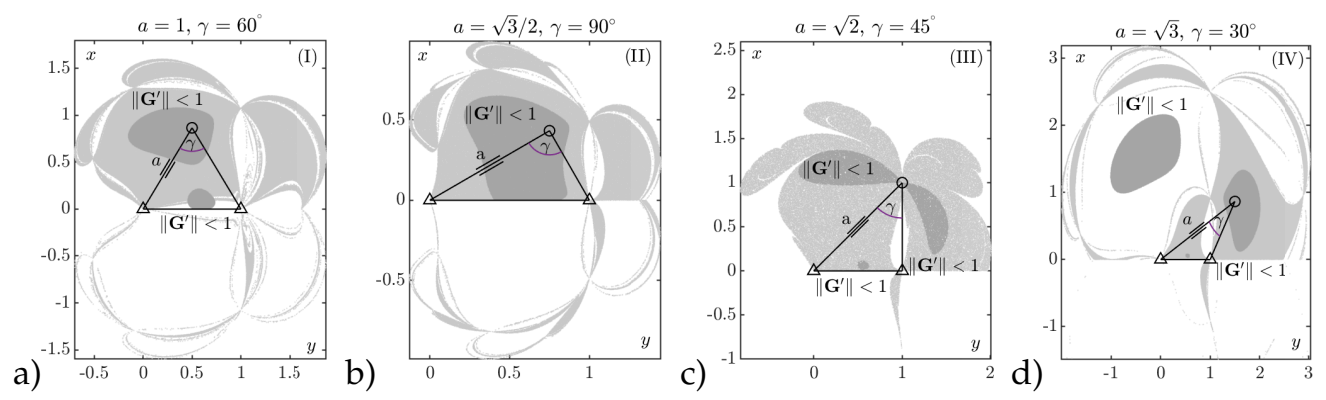

Figure 28. Areas $\left\|\mathbf{G}^{\prime}\right\|_{2}<1$ of the initial points of the iterative Newton's method of combined intersections and resections.

The study of the eigenvalues $\left|\lambda_{\max }\right|$ and norms of the derivative $\mathbf{G}^{\prime}(\mathbf{x})$ of the projection of combined intersections and resections, indicates that there are shared parts of basins of attraction and sets of the initial points, for which the norm $\left\|\mathbf{G}^{\prime}(\mathbf{x})\right\|$ satisfies the condition: $\left\|\mathbf{G}^{\prime}(\mathbf{x})\right\|<1$ (Figure 28). Unlike the case of the angular intersections, these sets may not be entirely included in the basins of combined intersections and resections (Figures 28(a) and (d)). For $\gamma=45^{\circ}$ the area $\left\|\mathbf{G}^{\prime}(\mathbf{x})\right\|<1$ contracts as the solution point $\mathbf{x}^{*}$. is approached. The radius of the neighborhood containing convergent points quickly decreases. It may be shown that in point $\mathbf{x}^{*}(x=1, y=1)$, which is the intersection solution, the matrix $\left\|\mathbf{G}^{\prime}(\mathbf{x})\right\|$ of the derivative of the projection and its norm are undetermined. The obtained results confirm that the condition: $\left\|\mathbf{G}^{\prime}(\mathbf{x})\right\|<1$ is not necessary for achieving iterative convergence of the Newton's method.

\section{Summary and conclusions}

The results obtained within this research indicate that basins of attraction of the considered basic geodetic constructions are characterized by vast heterogeneity of geometric and iterative structures. By performing theoretical simulations we demonstrate that basins of polynomial linear intersections are theoretically unlimited half-planes 
determined by the straight line passing through the points of the base and the intersected point. The basins of attraction of radical linear intersections are characterized by a greater complexity of geometric structures and distributions of iterations. The structures complexity of the intersections increases with the increase of departure from the symmetric intersection. In general cases, they are disconnected and composed of many geometrically similar subsets - branches (the effect of the set of affine projections of variable values of coefficients determined by nonlinear functions of observation sets). The analysis indicates that basins of symmetric intersection in the direction of the $x$ axis are unlimited, while they are limited in the direction of the $y$ axis. Existence of unlimited basins is attractive from the computational point of view, because every initial point is convergent.

On the basis of the performed computations for asymmetric cases of intersections, we notice that for very large values of coordinates of the initial points of the Newton's method, the Jacobian matrices of the iterative systems are singular. It signifies limitedness of the basins of attraction. In the case of limited basins, the information concerning the range of values of initial point coordinates is valuable, especially in the case of existence of disturbances of the iterative process and controlling it. We also notice, that the initial points on the edges of the branches of the basin correspond to large values of the number of iterations and appropriately long time of convergence. The mixed convergence is observed here, i.e. linear far from the point of solution (the branch edge), the quicker the closer the iterative point is to the constant point - and finally quadratic.

The study on basins of attraction of asymmetric linear intersections for various resolutions of grids of the initial points, show similarity of the obtained geometric structures and distributions of iterations in a wide range of changes of spatial scales. Similarity of iterative structures causes that the initial points located far from the solution point may converge similarly quickly as the ones in the solution's vicinity. The difference of the number of iterations is related here with the necessity of performing snaps through between the embedded branches (subsets) of the basin of attraction: $B_{j}, j \gg 1$.

When studying the impact of changes of the shape of the intersections on the basins we show that discrete changes of the value of measurement of the distance $a$ for established unit lengths of the distance $b$ and base $c$ (family $b=c=1$ ), correspond to qualitatively different basins of attraction (different number of branches). Such changes are typical for the bifurcation known from the theory of non-linear dynamic systems. We also determine the potential areas of convergence of the Newton's method resulting from the condition applied to the spectral radius: $\left|\lambda_{\max }\right|<1$ (or norm $\left\|\mathbf{G}^{\prime}(\mathbf{x})\right\|<1$ ). The performed calculations indicate that the Newton's method is convergent for the initial points belonging to such sets. Since the sets are contained in the basins of attraction, the condition $\left|\lambda_{\max }\right|<1\left(\left\|\mathbf{G}^{\prime}(\mathbf{x})\right\|<1\right)$ is not necessary (in the case of the considered intersections) for ensuring iterative convergence of the Newton's method. This conclusion also concerns angular intersections and the combined intersections/resections.

The study on basins of angular and combined intersections show that they are limited and disconnected, i.e. they are composed of separate subsets. The more precise high-resolution analysis showed that there are similar subsets in their neighborhood. For this reason, hypothetically each of the considered basins of attraction is composed of an infinite number of limited subsets of converging of the initial points. The diameters of the areas of basins of the analyzed families of angular and combined intersections/resections may reach the value of a few units of the base length. They are larger than those resulting from the condition: $\left|\lambda_{\max }\right|<1\left(\left\|\mathbf{G}^{\prime}(\mathbf{x})\right\|<1\right)$. We also notice that they rise with the increase of the length of the distances. In the subsets of the main basins of angular intersections there are neighborhoods of intersected points, radiuses are also of the order of the base length. It gives the possibility of quick selection of the initial point. On the other hand, the basins of the combined intersections/resections for $\gamma \approx 45^{\circ}$ may even have very small neighborhoods of intersected point that include the divergent points. In the case of the angular intersections, continuous change of $\alpha$ and $\beta$ angles corresponds 
to basins, which geometric and iterative structures are similar to the structure of the symmetric intersection basin assumed as the reference one. Continuous change of $\gamma$ angle of the combined intersections and resections may lead to the effect of change of the structure resulting from the trigger property of the function of the observation system. Combined intersections and resections for angles $\gamma \approx 45^{\circ}$ correspond to basins of complex transitional structures, and the basin for $\gamma=45^{\circ}$ is composed of subsets appearing in basins before $\gamma<45^{\circ}$ and after switching $\gamma>45^{\circ}$. These effects are not observed in the case of angular intersections. The initial points of angular and combined intersections / resections located far from the solution point may converge as quickly as the ones in the close neighborhood. The initial points belonging to various disconnected subsets of intersection basins converge to the solution through a series of snaps through. They are not accidental but realized according to certain schemes. Therefore, we deal with convergence on a global scale, i.e. convergence realized on a disconnected set of the basin of attraction. Probably, this kind of convergence has not been formalized in the form of appropriate mathematical theorems yet. We notice that in these cases the basic assumptions of the convergence theorems are not satisfied. For example, balls $K\left(\mathbf{x}^{0}\right)$ of convergent initial points do not contain the solution point $\mathbf{x}^{*}$. The basic condition of contracting, which provides unambiguity of solutions (fixed point method) is not satisfied either. In the examples presented in the paper, the iterative Newton's function $\mathrm{G}$ does not project balls $K\left(\mathbf{x}^{0}\right)$ into themselves but converts them into disconnected sets.

Summarizing, the basins of attraction of the considered intersections/resections may be analyzed with respect to the shape of the grid determined by the linear or angular measurements; the assumed accuracy of the determined solution $\mathbf{x}^{*}$ (determining the local structure in the vicinity of $\mathbf{x}^{*}$ ) and the number of iterations. Due to the amount of parameters determining the basin, it is a multidimensional manifold. A 2D spatial set composed of convergent initial points is related with each set of parameters. Multidimensionality of the considered basins is related with a number of issues. Some of them are analyzed in this paper, others - which are only mentioned - may be subjected to a separate detailed research. Moreover, the results presented in this paper may constitute the basis of theoretical research of more complex nonlinear systems of observation equations.

\section{Appendix A. Derivation of the formula (17)}

Formula (17) is the result of solving the system of equations (15):

$$
\left\{\begin{array}{l}
f_{1}(x, y)=x^{2}+y^{2}-a^{2}=0 \\
f_{2}(x, y)=x^{2}+(c-y)^{2}-b^{2}=0
\end{array}, x>0\right.
$$

The solution is obtained by subtracting the second from the first system equation (15)

$$
\left(x^{2}+y^{2}-a^{2}\right)-\left(x^{2}+y^{2}-2 c y+c^{2}-b^{2}\right)=0
$$

from where we get the variable $y$ :

$$
y=\frac{a^{2}-b^{2}+c^{2}}{2 c}
$$

Substituting $y$ to the first equation

$$
x^{2}=a^{2}-y^{2}=(a-y)(a+y)=\left(\frac{b^{2}-(c-a)^{2}}{2 c}\right)\left(\frac{(c+a)^{2}-b^{2}}{2 c}\right)
$$

after ordering we get formula (17)

$$
x=\frac{\sqrt{(a+b-c)(a+b+c)(c+b-a)(c+a-b)}}{2 c} .
$$




\section{Appendix B. Derivation of the relation for the function $g(x)(20)$}

Relationships (18) and (19) for the iterative function $\mathbf{G}$ (x indicate that the function $g(x)$ has the form:

$$
g(x)=x-(c-y) \frac{f_{1}(x, y)}{2 c x}-y \frac{f_{2}(x, y)}{2 c x},
$$

where $f_{1}(x, y)$ and $f_{2}(x, y)$ are functions of system (15)

$$
\left\{\begin{array}{l}
f_{1}(x, y)=x^{2}+y^{2}-a^{2}=0 \\
f_{2}(x, y)=x^{2}+(c-y)^{2}-b^{2}=0
\end{array}, x>0\right.
$$

Substituting the equation of the system (15) and the known value of $y=\frac{a^{2}-b^{2}+c^{2}}{2 c}$ (17) into (A1) we get

$$
\begin{aligned}
g(x)= & \frac{x}{2}+\frac{2 a^{2} b^{2}+2 a^{2} c^{2}+2 b^{2} c^{2}-a^{4}-b^{4}-c^{4}}{8 c^{2} x}= \\
& \frac{x}{2}+\frac{\left.\left((a+b)^{2}-c^{2}\right)\right)\left(c^{2}-\left(a-b^{2}\right)\right)}{8 c^{2} x}
\end{aligned}
$$

and finally

$$
g(x)=\frac{x}{2}+\frac{(a+b-c)(a+b+c)(c-a+b)(c+a-b)}{8 c^{2} x}>0, \text { for } x>0 .
$$

Author Contributions: The authors have equally contributed to the writing, editing, and style of this paper. All authors have read and agreed to the published version of the manuscript.

Funding: This research is supported by the Faculty of Civil Engineering and Geodesy statutory research founds.

Conflicts of Interest: The authors declare no conflicts of interest.

\section{References}

1. Banach, S. Sur les opérations dans les ensembles abstraits et leur application aux équations intégrales Fundamenta Mathematicae, 1922, 3(1), 133-181.

2. Dennis, J. E. Jr., Schnabel, R. B. Numerical Methods for Unconstrained Optimization and Nonlinear Equations; A Series: Classics In Applied Mathematics, Prentice-Hall, Inc., 1983, 16.

3. Ghilani, C. D. Adjustment computations: Spatial data analysis; Wiley, 6 Eds., 2017.

4. Guckenheimer, J., Holmes, P. Nonlinear Oscillations, Dynamical Systems, and Bifurcations of Vector Fields. A Series: Applied Mathematical Sciences, Springer, New York, 1983, 42.

5. Kelley, C. T. Iterative methods for linear and nonlinear equations. SIAM, Philadelphia, 1995.

6. Kincaid, D., Cheney, W. Numerical Analysis: Mathematics of Scientific Computing, American Mathematical Society, 2002.

7. Ortega J. M. Numerical Analysis. Computer Science and Applied Mathematics: A Series: Monographs and Textbooks. Academic Press, New York, 1972.

8. Ortega J. M., Rheinboldt, W. C. Iterative Solutions of Nonlinear Equations in Several Variables. Academic Press, New York, 1970.

9. Ostrowski A. M. Solutions of Equations and Systems of Equations. Pure and Applied Mathematics: A Series of Monographs and Textbooks, 2 Eds., Elsevier 1966, 9.

10. Traub, J. F. Iterative Methods for the Solution of Equations; American Mathematical Soc., Prentice-Hall, Englewood Cliffs, NJ, 1964.

11. Uren, J, Price, W. F. Intersection and Resection, In: Surveying for Engineers, Macmillan Education Ltd, London, 1985, 188-196.

12. Nusse, H. E., Yorke, J. A. Dynamics: Numerical Explorations. A Series: Applied Mathematical Sciences, 1998.

13. Barnsley, M. Fractals everywhere. Morgan Kaufmann Publishers, 2 Eds., 2000.

14. Nielsen, A. A. Least Squares Adjustment: Linear and Nonlinear Weighted Regression Analysis; (Technical University of Denmark Applied Mathematics and Computer Science; National Space Institute); http://www2.imm.dtu.dk/pubdb/views/edoc_download. php/2804/pdf/imm2804.pdf.

15. Lothar G. Developments with respect to the automated processing and analysis of engineering survey data, Proceedings of the Applications of Geodesy to Engineering: Symposium No. 108, Stuttgart, Germany; 175-184.

16. Čepek A. The GNU GaMa Project - Adjustment of Geodetic Networks. Acta Polytechnica, 2002, 42(3), 26-30

17. Čepek A. GNU Gama. Available online: URL https://www.gnu.org/software/gama/, 2020. 
18. Siki Z. GeoEasy an open source project for surveying calculations. Geoinformatics FCE CTU, 2018, 17)(2), 1-8, DOI: https://doi.org/10.14311/gi.17.2.1.

19. Kroszczynski K., Winnicki I. The properties of strange attractor reconstructed from the time series of tropospheric mean temperature, in Proceedings of the 18th International Conference on Interactive Information and Processing Systems for Meteorology, Oceanography and Hydrology (IIPS), 2002, Orlando, FL. 